\title{
Genome-wide identification and expression analysis of SBP-like transcription factor genes in Moso Bamboo (Phyllostachys edulis)
}

Feng Pan ${ }^{1 \dagger}$, Yue Wang ${ }^{1 \dagger}$, Huanglong Liu', Min Wu' ${ }^{1}$, Wenyuan Chu', Danmei Chen ${ }^{1}$ and Yan Xiang ${ }^{1,2^{*}}$

\begin{abstract}
Background: The SQUAMOSA promoter binding protein-like (SPL) proteins are plant-specific transcription factors (TFs) that function in a variety of developmental processes including growth, flower development, and signal transduction. SPL proteins are encoded by a gene family, and these genes have been characterized in two model grass species, Zea mays and Oryza sativa. The SPL gene family has not been well studied in moso bamboo (Phyllostachys edulis), a woody grass species.

Results: We identified 32 putative PeSPL genes in the P. edulis genome. Phylogenetic analysis arranged the PeSPL protein sequences in eight groups. Similarly, phylogenetic analysis of the SBP-like and SBP proteins from rice and maize clustered them into eight groups analogous to those from $P$. edulis. Furthermore, the deduced PeSPL proteins in each group contained very similar conserved sequence motifs. Our analyses indicate that the PeSPL genes experienced a large-scale duplication event 15 million years ago (MYA), and that divergence between the PeSPL and OsSPL genes occurred 34 MYA. The stress-response expression profiles and tissue-specificity of the putative PeSPL gene promoter regions showed that SPL genes in moso bamboo have potential biological functions in stress resistance as well as in growth and development. We therefore examined PeSPL gene expression in response to different plant hormone and drought (polyethylene glycol-6000; PEG) treatments to mimic biotic and abiotic stresses. Expression of three (PeSPL10, $-12,-17)$, six (PeSPL1, - 10,-12,-17,-20,-31), and nine (PeSPL5, -8, -9, -14,-15,-19,-20,-31, -32) genes remained relatively stable after treating with salicylic acid (SA), gibberellic acid (GA), and PEG, respectively, while the expression patterns of other genes changed. In addition, analysis of tissue-specific expression of the moso bamboo SPL genes during development showed differences in their spatiotemporal expression patterns, and many were expressed at high levels in flowers and leaves.
\end{abstract}

Conclusions: The PeSPL genes play important roles in plant growth and development, including responses to stresses, and most of the genes are expressed in different tissues. Our study provides a comprehensive understanding of the PeSPL gene family and may enable future studies on the function and evolution of SPL genes in moso bamboo.

Keywords: Moso bamboo, SPL genes, Transcription factor, Expression patterns

\footnotetext{
* Correspondence: xiangyanahau@sina.com

${ }^{\dagger}$ Equal contributors

${ }^{1}$ Laboratory of Modern Biotechnology, School of Forestry and Landscape

Architecture, Anhui Agricultural University, Hefei 230036, China

${ }^{2}$ Key Laboratory of Crop Biology of Anhui Province, School of Life Sciences,

Anhui Agricultural University, Hefei 230036, China
} 


\section{Background}

Moso bamboo (Phyllostachys edulis), a large woody bamboo species that is widely distributed in Asia, possesses great economic, ecological, and cultural values. $P$. edulis accounts for almost $70 \%$ of the total area dedicated to bamboo cultivation in China, and is used in the manufacture of paper, timber, art wares, and also as a food resource [1]. As a perennial plant, moso bamboo is usually characterized by rapid growth and a long vegetative period before flowering [2]. Because SPL genes are known to regulate inflorescence branching and kernel development along with other major physiological processes, it is important to probe the underlying function of moso bamboo SPL (PeSPL) genes to understand the regulation of flowering in bamboo.

In addition, transcriptional control depends on TFs, which can regulate gene expression in response to stresses such as drought, cold, and salinity, as well as pathogen stimuli and phytohormones. Thus, TFs are involved in regulating defense responses and gene regulation networks in plant growth and development. The SPL genes encode a special family of TFs that are unique to plants [3]. SPL proteins contain a highly conserved DNAbinding domain, the SBP domain, which consists of approximately 79 amino acid residues that include ten conserved cysteine and histidine residues. The SBP domain comprises two zinc-binding sites and individual DNAbinding and nuclear localization domains $[4,5]$. The two SPL genes (AmSBP1 and AmSBP2) first isolated from the snapdragon, Antirrhinum majus, were identified based on their direct interaction with a promoter sequence motif in SQUAMOSA, a floral meristem identity gene [4]. The first SPL gene identified in Arabidopsis was SPL3, which was shown to promote flowering under long day conditions. In addition, AtSPL3 binds to a conserved cis-element in the promoter region of $A P E T A L A 1$, a floral meristem identity gene that is an ortholog of $S Q U A$, similar to the snapdragon genes $A m S B P 1$ and $A m S B P 2$ [6].

Previous biochemical and physiological studies have demonstrated that the SPL family of TFs have positive functions in controlling plant development. In Arabidopsis, all 16 SPL genes in the genome have been identified [7], and they play essential roles in leaf development [8], flower development [9], shoot development [10], sporogenesis [11], fertility [12], reproductive stage and nutritional changes [13], plant hormone signal transduction [14], and leaf primordia interval formation [15]. The SPL genes are also involved in the regulation of copper homeostasis [16] and the gibberellic acid (GA) response [17]. At present, the SPL gene family has been isolated, identified, and characterized in a number of plant species including silver birch [18], green alga [19], rice [20], moss [21], maize [22], Populus trichocarpa [23], tomato [24], grape [25], apple [26], melons [27], Salvia miltiorrhiza
[28], castor bean [29], Gossypium hirsutum [30], Prunus mume [31], petunia [32], citrus [33], peanut [34], chrysanthemum [35], and pepper [36]. However, the SPL proteins in moso bamboo have not been studied, and their functions are unclear at present.

With the rapid development of high-throughput DNA sequencing technologies, genome sequencing has provided us with an opportunity to perform a genome-wide analysis of the SPL genes in moso bamboo. In this study, we conducted the first systematic, comprehensive analysis of the SPL genes in Phyllostachys edulis. We identified 32 putative PeSPL transcription factor genes, and systematically analyzed their structure, phylogenetic relationships, predicted conserved motifs, evolutionary patterns and divergence, cis-elements, and expression levels in response to different treatments and in five different organs.

\section{Methods}

\section{Database searches for moso bamboo SPL genes}

To identify the SPL gene members in moso bamboo, the Hidden Markov Model (HMM) profiles of all sequences containing an SBP domain (PF03110) were used to search the National Center for Gene Research database (http:// www.ncgr.ac.cn/bamboo) [37]. In addition, in order to verify the identity of these putative PeSPLs, the nonredundant candidate SPL genes in moso bamboo were identified using the InterproScan program to confirm the existence of the conserved SBP domain, and all of the SBPlike genes without an SBP domain were discarded [38]. Information for the PeSPL genes and predicted proteins, including CDS lengths, the predicted number of amino acids, and physicochemical parameters were obtained from the Bamboo GDB (http://www.bamboogdb.org).

\section{Phylogenetic analyses and intron-exon structure determination}

To examine the domain organization of the predicted SBP proteins in moso bamboo in detail, multiple sequence alignments of SBP domain-containing sequences were performed using Clustal W software [39], and we constructed a phylogenetic tree based on the complete PeSPL sequences using the N-J method as implemented in MEGA software (v5.1) [40] with a bootstrap analysis of 1000 replicates. The combined phylogenetic tree of OsSPL, ZmSPL and PeSPL protein sequences was generated using the same method. In addition, the predicted exon-intron structures of the PeSPL genes were visualized using the online Gene Structures Display Server (http://gsds.cbi.pku.edu.cn) by comparing the cDNA with the corresponding genomic DNA sequences.

\section{Identification of conserved protein sequence motifs}

Conserved motifs present in the PeSPL proteins were identified with the online MEME tool (http://meme.sdsc.edu/ 
meme/intro.html) using the default parameter settings: maximum number of motifs $=20$; optimum motif length range between 6 and 200 [41]. We also used the Pfam (http://pfam.sanger.ac.uk/search) [42] and SMART (http:// smart.embl-heidelberg.de/) databases to annotate the structural motifs [43].

\section{Identification of paralogs and rice orthologs in moso bamboo}

We used BLASTn $[44,45]$ to perform all-against-all nucleotide sequence similarity searches of the transcribed SPL sequences to identify paralogous sequences as shown by Blanc and Wolfe [44]. Sequences that showed at least $40 \%$ identity with aligned regions $>300$ bp were defined as paralogs. Putative rice orthologs were identified by using each sequence as a query to search against all sequences from moso bamboo. If the SPL gene sequences gave the best hit, and $>300 \mathrm{bp}$ of the two sequences aligned, the two genes were then defined as being orthologous [46].

\section{Calculation of $\mathrm{Ka} / \mathrm{Ks}$ values}

Pairwise alignments of the paralogous and orthologous SPL gene sequence pairs were performed with ClustalX 2.11, and the results were further analyzed using MEGA 6.0. A synonymous substitution (Ks) is defined as a mutation in which a nucleotide base is replaced by a different base in a protein-coding region of a gene that does not result in an amino acid change in the encoded protein, while a non-synonymous substitution (Ka) results in a change in the amino acid sequence of a protein [47]. The non-synonymous and synonymous substitution rates were then calculated using DnaSP 5 to analyze gene duplication events $[48,49]$. As described by Lin et al. (2014) Ks can be used as a proxy for time when dating large-scale duplication events [50]. Therefore, the date of duplication events was subsequently converted into divergence time ( $\mathrm{T}$ ) using the formula $\mathrm{T}=\mathrm{Ks} /$ $2 \lambda \times 10^{-6}$ Mya for each gene pair. Based on previous studies, the approximate value of the clock-like synonymous substitution rate $(\lambda)$ was $6.5 \times 10^{-9}$ years for both moso bamboo and rice $[37,51]$.

\section{Analysis of the putative promoter regions of the PeSPL genes}

To identify the cis-elements in the putative promoter regions, we examined the $2000 \mathrm{bp}$ upstream sequences of the PeSPL genes. We used the PLACE website (http:// www.dna.affrc.go.jp/PLACE/signalscan.html) [52] and Plant-CARE (http://bioinformatics.psb.ugent.be/webtools /plantcare/html/) [53] to identify the predicted cis-regulatory elements present in the gene promoters.

\section{Plant growth conditions and seedling treatments}

The treatment-induced gene expression profiles of 32 PeSPL genes were examined in young leaves of threemonth old seedlings of moso bamboo grown from seeds collected from Guilin in Guang Xi Province, China. All seeds were provided and identified by the Guilin Forestry Bureau. The moso bamboo seeds were germinated in culture dishes on moist, sterile filter paper at $25{ }^{\circ} \mathrm{C}$ in the dark. The seedlings were then moved into plastic pots containing a mixture of black soil and vermiculite and grown in a greenhouse under $14 \mathrm{~h}$ of light (from $07: 30$ to $21: 30$ ) at $24-28{ }^{\circ} \mathrm{C}$ and $80 \%$ humidity. These seedlings were then used in experiments to assay gene expression in response to three stress conditions. The moso bamboo seedlings were watered with Hoagland's nutrient solution twice a week. The treatments were performed by spraying the young moso bamboo leaves individually with $100 \mu \mathrm{M}$ gibberellin (GA), 20\% polyethylene glycol-6000 (PEG) solution, and $100 \mu \mathrm{M}$ salicylic acid (SA). The young leaves from the stress-treated plants were collected at $1,3,6,12$, and $24 \mathrm{~h}$ after treatment. Untreated seedlings were used as the control groups. Also, tissue-specific transcription profiles of $32 \mathrm{PeSPL}$ genes were analyzed in various vegetative and reproductive tissues in plants collected from Ningguo, Anhui Province, China. In addition, the permission of tissues collection and identification for the experiments were obtained from Jianguo Pei of Ningguo Forestry Bureau. The samples included various tissues (young leaves, mature leaves, roots, shoots, and panicles). After gathering, the plant tissues were immediately frozen in liquid $\mathrm{N}_{2}$ and stored at $-80{ }^{\circ} \mathrm{C}$ prior to RNA extraction.

\section{Microarray-based expression analysis}

We performed a comprehensive expression profile of the PeSPL genes in order to reveal their function in moso bamboo growth and development. The data was obtained from the NCBI Short Read Archive (SRA) database. The unprocessed RNA-seq reads from BioProject ERP001341 were then pruned to eliminate low quality base-calls $(\mathrm{Q}<20)$ and adaptor sequences using the pipeline Fastq clean [54]. The clean paired reads were mapped to the Phyllostachys edulis reference genome using the pipeline tophat 2 with the default parameters. Briefly, TopHat 2 uses Bowtie 2 as an alignment 'engine' and breaks reads that Bowtie 2 can not align on its own into segments [55]. And then, the differences in gene expression were tested with Cufflinks [55]. The heatmap of PeSPL gene expression was drawn with the Heatmapper Plus tool (http://www.bar.utoronto.ca/ntools/cgi-bin/ ntools_heatmapper.cgi) for seven moso bamboo tissues and different developmental stages (leaf, early panicle, advanced panicle, root, rhizome, 20-cm shoot, and 50cm shoot) [56]. 


\section{RNA isolation and qRT-PCR analysis}

Total RNA was extracted from frozen young leaf tissue from different stress treatments and different organs of moso bamboo with TRIzol reagent (Invitrogen, Ca, USA) as directed by the manufacturer. We synthesized firststrand cDNA using the Prime-Script ${ }^{\text {tax }}$ RT Reagent Kit (TaKaRa) according to the manufacturer's instructions. We then designed 32 pairs of gene-specific primers using Primer Express 3.0. Primer specificity was checked by BLAST searches using data from a local CDS database downloaded from BambooGDB (http://www.bamboogdb.org/page/microrna.jsp). In this study, the TIP41 (tonoplast intrinsic protein 41) gene was used as a reference for normalization because it has a stable expression pattern [57]. qRT-PCR amplifications were performed on an ABI 7300 Real-Time system (Applied Biosystems) in $20 \mu \mathrm{l}$ reactions containing $1 \mu \mathrm{l}$ of each gene-specific primer, $1 \mu \mathrm{l}$

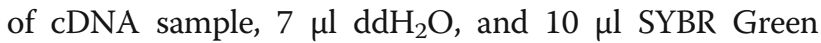
Master Mix reagent (Applied Biosystems). All primers for amplification of PeSPL genes are given in Additional file 1: Table S1. The qRT-PCR amplification conditions were: $95{ }^{\circ} \mathrm{C}$ for $30 \mathrm{~s}$, followed by 40 cycles of $95^{\circ} \mathrm{C}$ for $10 \mathrm{~s}, 55^{\circ}$ $\mathrm{C}$ for $15 \mathrm{~s}$, and $72{ }^{\circ} \mathrm{C}$ for $10 \mathrm{~s}$. A melting curve analysis was performed for each sample to verify the specificity of the reactions. There were three biological and three technical replicates performed for each sample. The relative expression levels were evaluated using the $\Delta \Delta C T$ method. It is worth noting that for the stress treatments, relative gene expression $\left[2^{-\Delta \Delta C T, C K}\left(\begin{array}{ll}0 & h\end{array}\right]\right.$ for each gene in the control plants was normalized to 1 as described previously [58].

\section{Results}

Identification and characterization of SPL family genes in moso bamboo

In this study, we identified 32 SPL genes in moso bamboo after removing redundant sequences from further analysis. We named the $32 \mathrm{SPL}$ genes PeSPL1 to PeSPL32 based on their chromosomal locations from top to bottom. All of these sequences were located, and the details of the SPL gene family in moso bamboo is given in Table 1 . The predicted PeSPL proteins varied greatly with respect to molecular weight and length. The moso bamboo SPL genes encode proteins ranging from 177 (PeSPL18) to 1071 (PeSPL30) amino acids (aa) in length, and from 18,838.3 (PeSPL18) to 118,663 (PeSPL30) $\mathrm{kDa}$ in molecular weight. The other characteristics of the individual SPL genes, including isoelectric point (pI) and the number of predicted exons, are also given in Table 1.

\section{Phylogenetic analysis of the PeSPL genes}

The functions of some SPL genes have been characterized in rice (Oryza sativa), a model species in the botanical family Poaceae. To examine the inferred evolutionary relationships between the SBP domain-containing proteins in the grass family, we constructed a phylogenetic tree from alignments of the full-length SPL protein sequences for members of three major Poaceae subfamilies; the Bambusoideae (Phyllostachys edulis), Ehrhartoideae (rice), and Panicoideae (Zea mays) (Fig. 1). The OsSPL and ZmSBP sequences were obtained, and we generated a phylogenetic tree using the Neighbor-joining (NJ) algorithm in MEGA software (v5.1) with the PeSPL sequences. The phylogenetic tree contains 82 putative SPL protein sequences from the three monocot species; 18 from the Oryza sativa, 32 from Zea mays, and 32 from P. edulis. The detailed characteristics of the SPL genes from rice and maize are given in Additional file 1: Table S2. The phylogenetic tree showed that the predicted SPL proteins clustered into eight groups, G1- G8, as described previously (Fig. 1).

\section{Determination of exon-intron structure and identification} of conserved sequence motifs in moso bamboo SPL genes We examined the structural diversity of the SPL gene family in moso bamboo by constructing a phylogenetic tree based on the full-length predicted PeSPL protein sequences. This analysis also grouped these proteins into eight clusters, and agrees well with the description given above for the three plant species (Figs. 1 and 2a). A possible mechanism driving the evolution of multigene families involves genetic structural diversity. We compared the numbers, lengths, and arrangement of the exons and introns in the gene sequences (Fig. 2b) to gain further insight into the structural diversity of the PeSPL genes. As shown in Fig. 2, the genes in group 7, group 8, and PeSPL19 in group 6 contain the largest number of exons (11). The other 26 genes contain between 1 and 7 exons. The results also show that PeSPL13 in group 3 has no introns or upstream sequences. In addition, the PeSPL11 gene in group 4 has no downstream sequence. An interesting feature of this analysis is that five sister gene pairs (PeSPL30/-14, PeSPL17/-2, PeSPL31/-5, PeSPL25/-6 and PeSPL4/-24) were found to have the same intron phase and intron/exon number. However, the intron lengths showed considerable length variation in these five sister gene pairs. We also identified some differences; for example, eight sister gene pairs (PeSPL20/-19, PeSPL13/-7, PeSPL32/-10, PeSPL29/-16, PeSPL28/-1, PeSPL12/-22, PeSPL21/-23 and PeSPL8/-9) varied greatly with respect to their structural organization and the numbers of introns and exons.

We initially searched for conserved sequence motifs using the MEME web server to further understand the compositions and diversity of motifs present in the predicted PeSPL proteins. A total of 20 distinct motifs were identified and designated motif 1 to motif 20 (Fig. 3); the details of the conserved amino acid sequences and their 
Table 1 Gene names, genomic information, and predicted protein parameters for the 32 putative PeSPL genes in the moso bamboo genome

\begin{tabular}{|c|c|c|c|c|c|c|c|c|}
\hline \multirow[t]{2}{*}{ Name } & \multirow[t]{2}{*}{ Gene ID } & \multirow[t]{2}{*}{ Chr } & \multirow[t]{2}{*}{ Location } & \multirow{2}{*}{$\begin{array}{l}\text { CDS } \\
\text { length(bp) }\end{array}$} & \multicolumn{3}{|l|}{ Protein } & \multirow[t]{2}{*}{ Exons } \\
\hline & & & & & Size (aa) & $\mathrm{MW}(\mathrm{kDa})$ & pl & \\
\hline PeSPL1 & PH01000002G1660 & PH01000002 & PH01000002:1,200,472-1,205,728(+ stand) & 1278 & 425 & $46,157.1$ & 8.45 & 4 \\
\hline PeSPL2 & PH01000040G1230 & PH01000040 & PH01000040:816,810-825,960(+ stand) & 2850 & 949 & 103,586 & 5.73 & 11 \\
\hline PeSPL3 & PH01000050G0170 & PH01000050 & PH01000050:85,280-89,502(+ stand) & 1080 & 359 & $38,147.5$ & 8.99 & 4 \\
\hline PeSPL4 & PH01000057G0060 & PH01000057 & PH01000057:47,613-53,685(+ stand) & 1044 & 347 & $38,395.5$ & 8.39 & 3 \\
\hline PeSPL5 & PH01000095G1560 & PH01000095 & PH01000095:1,058,556-1,062,174(+ stand) & 987 & 328 & $35,561.9$ & 8.63 & 4 \\
\hline PeSPL6 & PH01000117G1390 & PH01000117 & PH01000117:964,573-972,149(- stand) & 1236 & 411 & $43,399.7$ & 7.97 & 3 \\
\hline PeSPL7 & PH01000150G0460 & PH01000150 & PH01000150:291,251-295,678(+ stand) & 1071 & 356 & $36,355.1$ & 7.24 & 3 \\
\hline PeSPL8 & PH01000164G0630 & PH01000164 & PH01000164:351,140-355,432(+ stand) & 1389 & 463 & $50,159.3$ & 9.42 & 4 \\
\hline PeSPL9 & PH01000176G0670 & PH01000176 & PH01000176:478,695-482,876(- stand) & 1455 & 484 & $52,719.5$ & 9.31 & 5 \\
\hline PeSPL10 & PH01000450G0480 & PH01000450 & PH01000450:319,332-324,328(+ stand) & 1572 & 523 & $56,864.4$ & 8.84 & 7 \\
\hline PeSPL11 & PH01000548G0610 & PH01000548 & PH01000548:433,324-435,597(- stand) & 912 & 303 & $32,689.6$ & 6.61 & 3 \\
\hline PeSPL12 & PH01000608G0740 & PH01000608 & PH01000608:478,612-483,867(+ stand) & 1287 & 428 & $45,044.4$ & 7.68 & 4 \\
\hline PeSPL13 & PH01000770G0270 & PH01000770 & PH01000770:236,104-237,441(+ stand) & 939 & 312 & $34,173.7$ & 9.09 & 1 \\
\hline PeSPL14 & PH01000915G0320 & PH01000915 & PH01000915:189,707-197,542(- stand) & 3186 & 1061 & 116,841 & 6.91 & 11 \\
\hline PeSPL15 & PH01000920G0420 & PH01000920 & PH01000920:317,552-324,017(+ stand) & 2076 & 691 & $76,451.9$ & 5.85 & 11 \\
\hline PeSPL16 & PH01000969G0180 & PH01000969 & PH01000969:168,903-175,012(+ stand) & 879 & 292 & $32,015.7$ & 6.35 & 3 \\
\hline PeSPL17 & PH01000985G0610 & PH01000985 & PH01000985:379,393-386,488(- stand) & 2865 & 954 & 104,628 & 5.53 & 11 \\
\hline PeSPL18 & PH01001167G0360 & PH01001167 & PH01001167:221,659-223,521(- stand) & 534 & 177 & $18,838.3$ & 8.61 & 2 \\
\hline PeSPL19 & PH01001316G0020 & PH01001316 & PH01001316:5985-15,805(- stand) & 2427 & 808 & $88,965.7$ & 5.34 & 11 \\
\hline PeSPL20 & PH01001685G0440 & PH01001685 & PH01001685:285,382-288,941(- stand) & 585 & 194 & $21,258.3$ & 8.57 & 3 \\
\hline PeSPL21 & PH01001751G0380 & PH01001751 & PH01001751:279,930-283,848(- stand) & 1008 & 335 & $36,542.2$ & 9.05 & 4 \\
\hline PeSPL22 & PH01001834G0370 & PH01001834 & PH01001834:290,182-294,145(- stand) & 1236 & 411 & $43,721.6$ & 8.73 & 3 \\
\hline PeSPL23 & PH01001853G0340 & PH01001853 & PH01001853:289,935-292,481(- stand) & 969 & 322 & $34,303.1$ & 8.56 & 3 \\
\hline PeSPL24 & PH01001910G0100 & PH01001910 & PH01001910:76,552-80,372(+ stand) & 1044 & 347 & $38,418.6$ & 8.73 & 3 \\
\hline PeSPL25 & PH01002673G0070 & PH01002673 & PH01002673:47,490-53,632(+ stand) & 1203 & 400 & $42,694.8$ & 7.57 & 3 \\
\hline PeSPL26 & PH01002789G0180 & PH01002789 & PH01002789:101,788-107,846(- stand) & 966 & 321 & $34,363.5$ & 6.93 & 3 \\
\hline PeSPL27 & PH01003044G0090 & PH01003044 & PH01003044:55,489-58,672(+ stand) & 585 & 195 & $20,422.9$ & 9.62 & 2 \\
\hline PeSPL28 & PH01003178G0220 & PH01003178 & PH01003178:102,673-108,004(- stand) & 1155 & 384 & $41,253.5$ & 9.44 & 3 \\
\hline PeSPL29 & PH01003773G0220 & PH01003773 & PH01003773:1 15,930-124,043(+ stand) & 1284 & 427 & $45,833.1$ & 8.59 & 4 \\
\hline PeSPL30 & PH01004096G0180 & PH01004096 & PH01004096:90,610-97,402(- stand) & 3216 & 1071 & 118,663 & 6.13 & 11 \\
\hline PeSPL31 & PH01004816G0090 & PH01004816 & PH01004816:49,108-52,984(+ stand) & 993 & 331 & $35,967.5$ & 8.96 & 4 \\
\hline PeSPL32 & PH01007654G0010 & PH01007654 & PH01007654:454-3458(+ stand) & 963 & 320 & $35,200.1$ & 8.92 & 5 \\
\hline
\end{tabular}

lengths are shown in Additional file 1: Table S3. It is clear that some of the gene pairs (PeSPL4/-24, PeSPL25/-6, PeSPL31/-5, PeSPL8/-9, PeSPL6/-29, PeSPL25/-29) share complete motif profiles (Fig. 3), and that the sister gene pairs in general contain very similar conserved structural motifs. In addition, it is very interesting that some motifs were found to be specific to only one or two groups of PeSPL proteins. For instance, motifs 9 and 10 are found exclusively in group G2, and motif 19 is only present in group G3.

\section{Patterns of evolution and divergence in the SPL gene family between moso bamboo and rice}

Our analysis identified 16 putative paralogous gene pairs $(\mathrm{Pe}-\mathrm{Pe})$ in the moso bamboo genome and 21 ortholog pairs $(\mathrm{Pe}-\mathrm{Os})$ between the PeSPL and OsSPL genes using BLASTn sequence similarity analyses. All of the paralogous and orthologous pairs are listed in Table 2. For every SPL gene pair, we calculated the $\mathrm{Ka} / \mathrm{Ks}$ ratios to estimate divergence times for the duplicated SPL genes (see Additional file 1: Tables S4 and S5). To further 


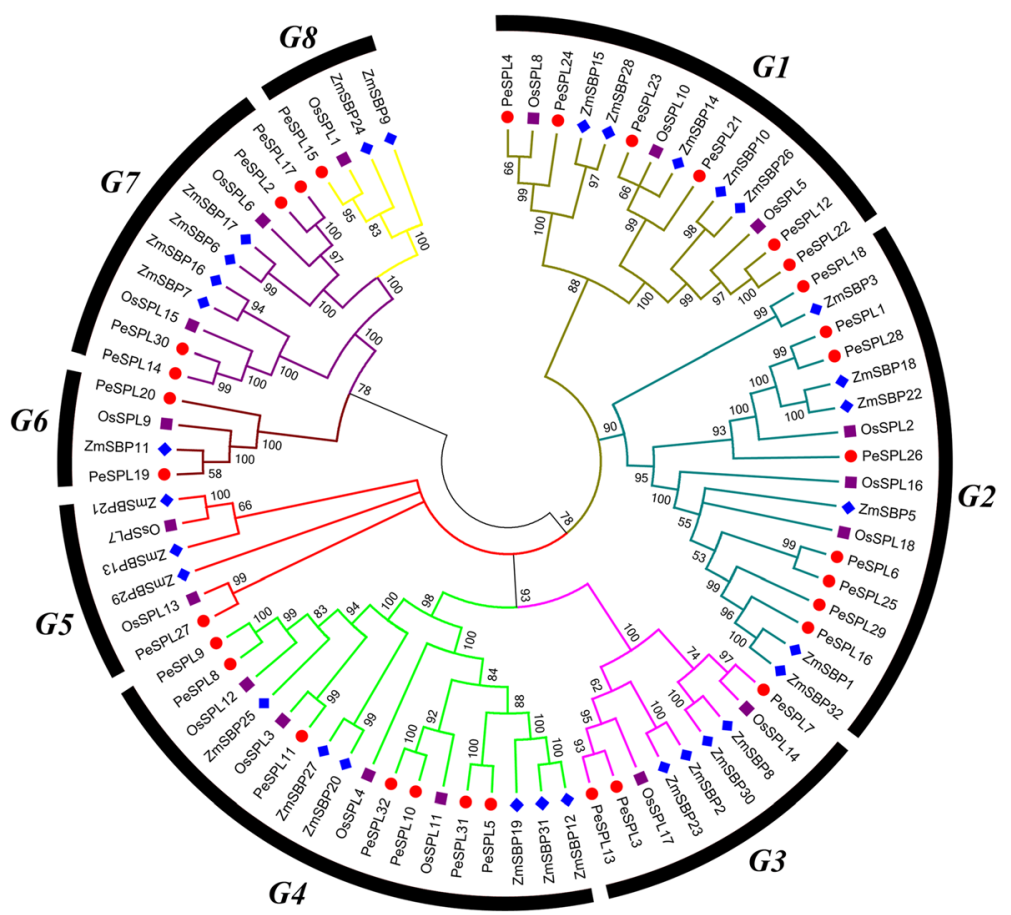

Fig. 1 Phylogeny and distribution of SPL protein from three plant species. Phylogenetic tree of SPL proteins from rice, maize and moso bamboo. The tree was generated with Clustal X 2.0 software using the neighbour-joining method

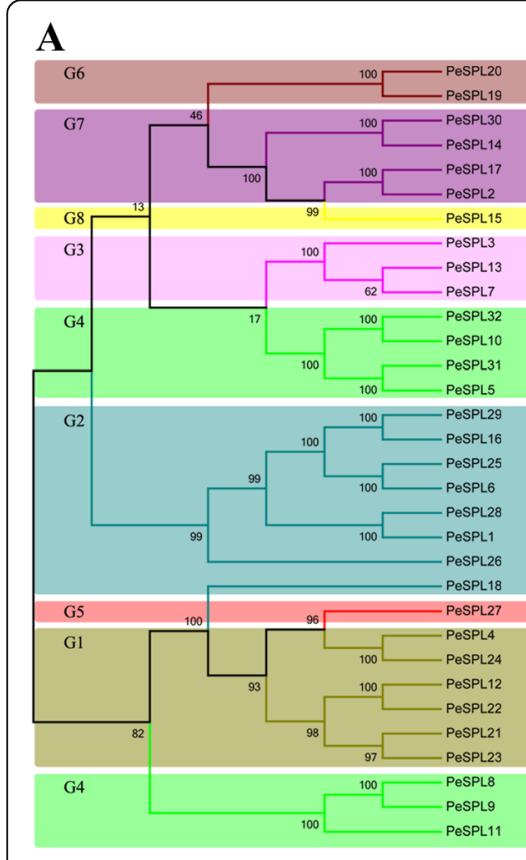

\section{B}

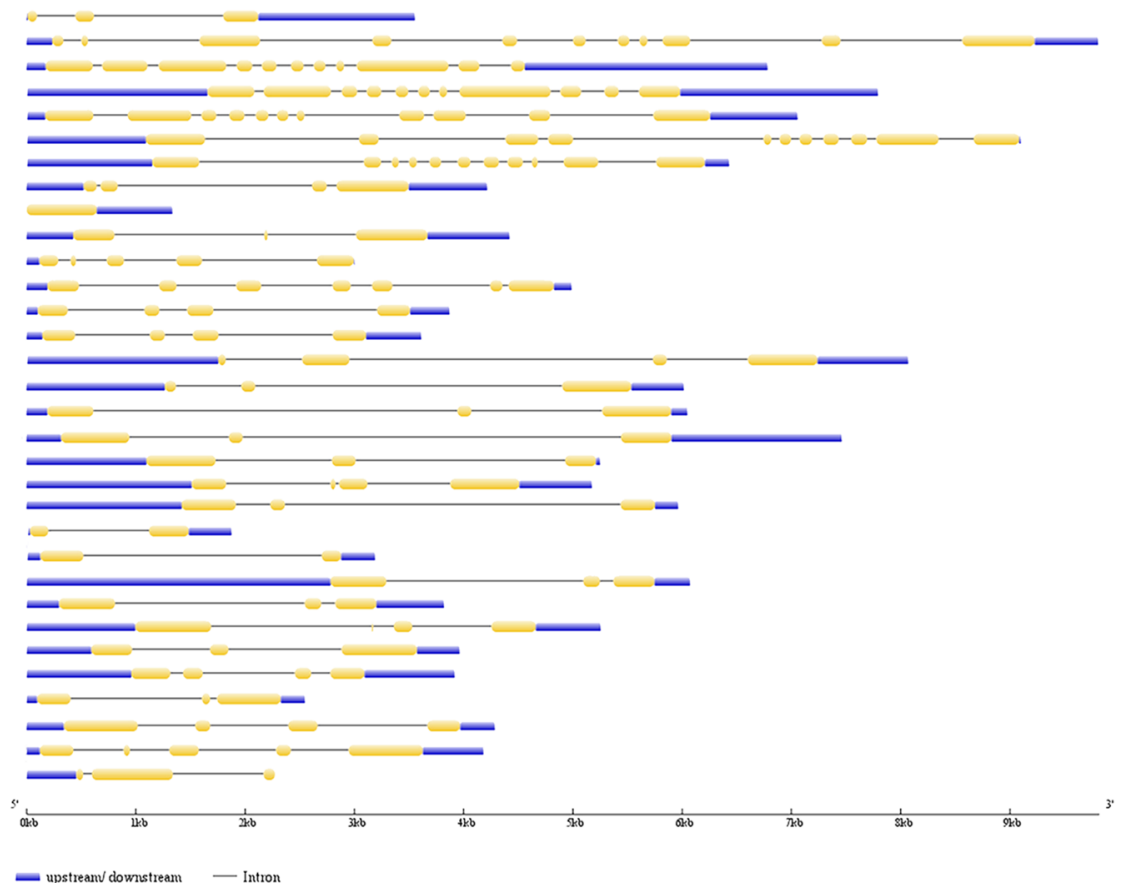

Fig. 2 The unrooted tree of PeSPL genes family and exons-introns organization of moso bamboo (Phyllostachys edulis) SPL proteins. a The number of above or below branches of the phylogenetic tree indicate bootstrap values. Bootstrap values from 1000 replicates are indicated at each node. The unrooted tree was constructed with Clustal X2.0 by the Neighbor-Joining (NJ) method with 1000 bootstrap replicates. The middle set of numerals on the tree represented eight PeSPL groups. $\mathbf{b}$ Yellow rectangles represent exons, grey lines represent introns and blue boxes represent Untranslated regions (UTRs) 


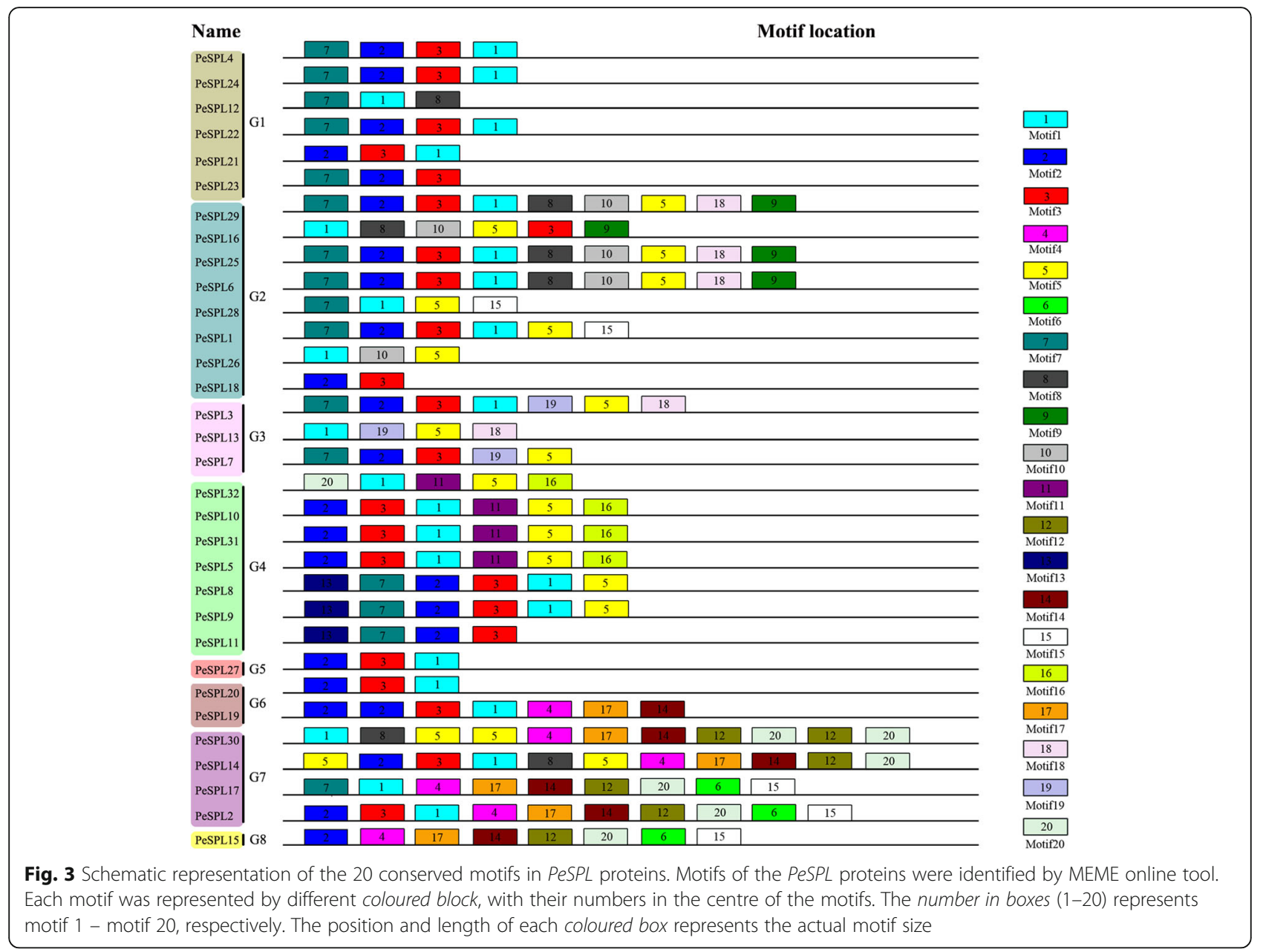

study divergence times between rice and moso bamboo, we also performed a statistical analysis of the $\mathrm{Ka} / \mathrm{Ks}$ ratios and the Ks values. The frequency distributions of the $\mathrm{Ka} / \mathrm{Ks}$ and $\mathrm{Ks}$ values for the orthologous and paralogous gene pairs from the rice and moso bamboo genomes were calculated (Fig. 4). The distribution of the calculated Ks values for the paralogous pairs in moso bamboo averaged $\sim 0.2$ (Fig. 4a), indicating that the SPL genes experienced a large-scale duplication event approximately 15 million years ago (MYA). A previous study estimated the timing of a whole-genome duplication in moso bamboo at 7-12 MYA [37], which could indicate that the large-scale duplication of the SPL genes occurred earlier. Also, for the rice-moso bamboo orthologous pairs, the major peak at 0.45 (Fig. 4b) shows that for the SPL genes the divergence time is 34 MYA. A comparison with the previous study that concluded that the divergence time between moso bamboo and rice was 7-15 MYA indicates that the SPL genes underwent gene evolution prior to the separation of the two progenitor species [59]. The $\mathrm{Ka} / \mathrm{Ks}$ peaks in the moso bamboo genome are distributed between $0.2-0.3$ (Fig. 4c), while the
$\mathrm{Ka} / \mathrm{Ks}$ ratios between the rice and moso bamboo genomes are distributed at 0.4-0.5 (Fig. 4d) [59]. This suggests that there was strong purifying selection in the SPL genes between the moso bamboo and rice genomes, as well as for the paralogs in the moso bamboo genome.

\section{Analysis of putative promoter regions in the PeSPL gene family}

Gene expression patterns and/or tissue specificity in response to stress are largely determined by cis-regulatory elements [60], and cis-regulatory elements in the promoter regions are closely correlated with multi-stimulus responsive genes [61, 62]. Four types of cis-elements containing a dehydration-responsive element, a TCA element involved in regulation of SA-related genes, a gibberellic acid response element (GARE) motif, and tissue-specific and development-related elements (Additional file 1: Table S6) were detected in current studies. This leds us to search the promoter regions of the moso bamboo SPL genes for possible stress-responsive and tissue-specific ciselements using the PLACE and PlantCARE databases. Cis-elements can be located 2000-bp upstream of the 
Table 2 Paralogous ( $\mathrm{Pe}-\mathrm{Pe}$ ) and orthologous (Pe-Os) SPL gene pairs in moso bamboo and rice

\begin{tabular}{|c|c|}
\hline$P e-P e$ & $\mathrm{Pe}-\mathrm{Os}$ \\
\hline PeSPL1/PeSPL28 & PeSPL15/OsSPL1 \\
\hline PeSPL31/PeSPL5 & PeSPL9/OsSPL3 \\
\hline PeSPL30/PeSPL14 & PeSPL11/OsSPL3 \\
\hline PeSPL29/PeSPL6 & PeSPL31/OsSPL4 \\
\hline PeSPL29/PeSPL25 & PeSPL5/OsSPL4 \\
\hline PeSPL29/PeSPL16 & PeSPL2/OsSPL6 \\
\hline PeSPL23/PeSPL21 & PeSPL17/OsSPL6 \\
\hline PeSPL20/PeSPL19 & PeSPL24/OsSPL8 \\
\hline PeSPL17/PeSPL2 & PeSPL4/OsSPL8 \\
\hline PeSPL12/PeSPL22 & PeSPL19/OsSPL9 \\
\hline PeSPL10/PeSPL32 & PeSPL20/OsSPL9 \\
\hline PeSPL8/PESPL9 & PeSPL9/OsSPL12 \\
\hline PeSPL7/PeSPL13 & PeSPL8/OsSPL12 \\
\hline PeSPL6/PeSPL25 & PeSPL7/OsSPL 14 \\
\hline PeSPL4/PeSPL24 & PeSPL14/OsSPL15 \\
\hline \multirow[t]{6}{*}{ PeSPL3/PeSPL13 } & PeSPL30/OsSPL15 \\
\hline & PeSPL25/OsSPL 18 \\
\hline & PeSPL6/OsSPL18 \\
\hline & PeSPL29/OsSPL18 \\
\hline & PeSPL13/OsSPL17 \\
\hline & PeSPL3/OsSPL17 \\
\hline
\end{tabular}

promoter sequences and have practical impact on binding to target genes $[63,64]$. Therefore, we searched the 2000-bp upstream sequences to identify putative cisregulatory elements. We found many stress- and development-related elements in the promoter regions of the PeSPL genes. For example, there are $\sim 20$ drought-stress elements (S000415) in the PeSPL24 promoter region, and the PeSPL10 promoter contains as many as 12 tissue-specific elements. In addition, many genes have one or two SA and gibberellin responsive elements. Thus, further analysis of the putative promoter regions of PeSPL family genes helps to advance our understanding of stress tolerance and tissue-specificity in moso bamboo. Our results provide an indication that most SPL genes may function to enhance stress resistance, and are related to the developmental pathway.

\section{Differential expression profiling of SPL genes in moso bamboo tissues}

In order to study the dynamics of PeSPL gene expression, we examined the gene expression profiles in different organs using high-throughput RNA sequencing (RNA-seq), which is a next-generation sequencing technology that provides a snapshot of gene expression profiles and mRNA levels at a given time $[65,66]$. The
RNA-seq reads can be aligned to the draft genome sequence of moso bamboo [37]. Previous studies mainly focused on the expression profiles in different tissues [67-69]. However, the expression profiles of PeSPL genes are not well characterized at present.

We used microarray analysis to estimate the expression level of each PeSPL gene in different plant organs. The microarray data for 32 PeSPL genes (Additional file 1: Table S7) was downloaded from the NCBI database, and a heatmap was generated (Fig. 5).

The heatmap showed that six gene pairs (PeSPL2/ -17, PeSPL4/-24, PeSPL5/-31, PeSPL6/-25, PeSPL8/-9 and PeSPL14/-30) from the previously identified 16 paralogs in our study displayed distinct expression profiles in different developmental stages or different organs, revealing the different evolutionary fates of duplicated genes. For example, PeSPL25 was expressed at high levels in the early panicle (P1) and advanced panicle (P2), while its counterpart PeSPL6 had a relatively low expression level. However, the remaining 16 paralogous gene pairs had the same or similar relative expression levels. It is worth noting that 11 genes (PeSPl2, PeSPl3, PeSPl8, PeSPl9, PeSPl13, PeSPL14, PeSPL15, PeSPL17, PeSPL20, PeSPL27, and PeSPL30) showed high expression levels in these seven organs or developmental stages, suggesting that SPL genes play vital roles in plant development and growth.

\section{Differential expression profiling of PeSPL genes}

Plants often experience a variety of environmental stresses that affect their growth. Therefore, in our research, we examined the expression levels of all PeSPL genes under stress conditions to identify those that are involved in the responses to biotic and abiotic stress in moso bamboo. In a previous study, SA was reported to activate the transcription of many defense-related genes in response to pathogen infection [36]. In addition, SPL genes in Arabidopsis, such as SPL3 and SPL8, help mediate the response to GA signaling during plant development [17]. In addition, water content is a serious environmental stress that affects the growth of moso bamboo. When water resources are limited, plants reallocate this precious resource by restricting transpiration, and they frequently flower early [70]. It is wellknown that flowering will lead to death in moso bamboo. Thus, we used PEG to simulate drought conditions to observe its effect on the expression levels of PeSPL genes. In summary, we quantified the expression of the PeSPL genes in response to salicylic acid (SA), GA, and PEG (drought) treatments in moso bamboo using qRT-PCR.

Previous studies on SPL genes focused on plant growth and development by examining the relative expression in 
A

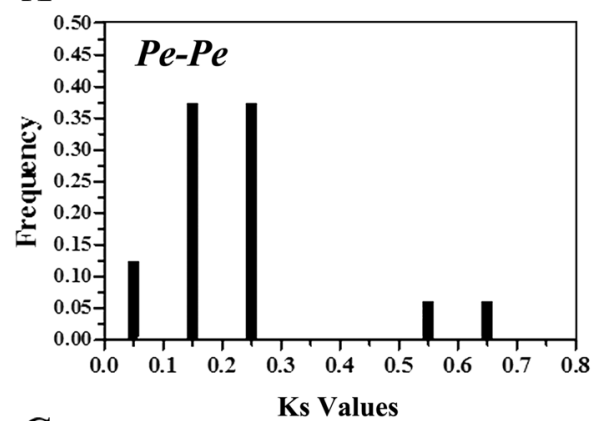

C

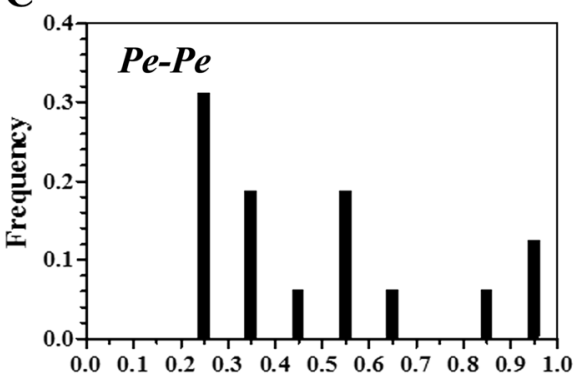

$\mathrm{Ka} / \mathrm{Ks}$ Values

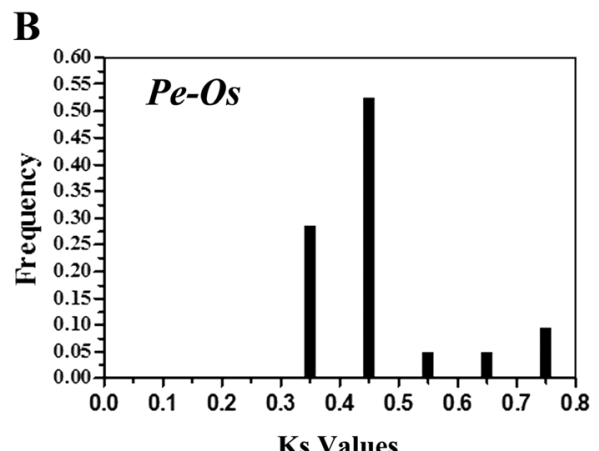

D

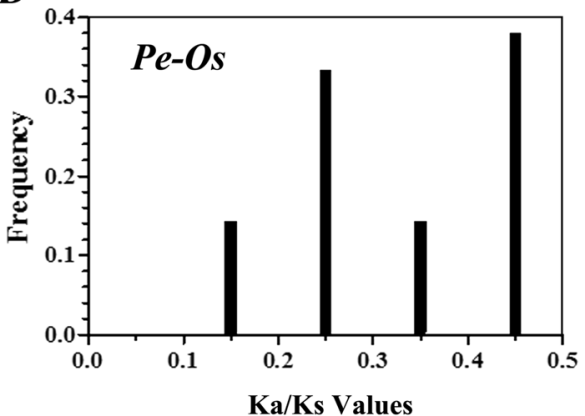

Fig. $4 \mathrm{Ks}$ and $\mathrm{Ka} / \mathrm{Ks}$ value distribution of the SPL genes in the genomes of moso bamboo paralogous gene-pairs (Pe-Pe) and orthologous genepairs between moso bamboo and rice viewed through the frequency distribution of relative Ks and Ka/Ks modes. Distribution of Ks and Ka/Ks values were obtained from paralogous gene-pairs ( $P e-P e)$ in the moso bamboo genome (a and $\mathbf{c}$ ), and orthologous gene-pairs between moso bamboo and rice (b and $\mathbf{d}$ )

different tissues and during several stages of plant growth. To investigate the roles of SPL genes in Phyllostachys edulis organs and development, we also performed qRTPCR analyses to examine the relative expression of the 32 PeSPL genes in young leaves, mature leaves, roots, shoots, and panicles.

In the SA treatment (Fig. 6), $30 \mathrm{PeSPL}$ family genes were found to be up-regulated; however, transcription of PeSPL5 and PeSPL19 was reduced at all time points. We found that PeSPL14 and PeSPL15 were highly expressed after $1 \mathrm{~h}$ of treatment, and the expression of PeSPL28 was highest at $6 \mathrm{~h}$. Expression of 19 genes (PeSPL3, -4, $-6,-7,-8,-9,-11,-13,-16,-21,-22,-23,-24,-25$, $-26,-27,-29,-30$, and 32) peaked at $24 \mathrm{~h}$. PeSPL7 showed the greatest up-regulation (by more than 230fold), and transcription of PeSPL21 increased $>110$-fold at $24 \mathrm{~h}$ after treatment. In addition, there were six duplicated gene pairs; PeSPL1 and -28, PeSPL4 and -24, PeSPL7 and -13, PeSPL8 and -9, PeSPL16 and -29, and PeSPL21 and -23, that had similar expression profiles in response to $\mathrm{SA}$ treatment.

In the GA treatments (Fig. 7), only three paralogous pairs, PeSPL1 and -28, PeSPL7 and -13, and PeSPL21 and -23 , displayed similar expression profiles. However, differential expression patterns were observed in some duplicated gene pairs. For example, in the paralogous pair PeSPL14 and -30, PeSPL14 showed only minor changes in expression ( 2 -fold higher than the control), while transcription of PeSPL30 increased nearly 100-fold over the check at $24 \mathrm{~h}$. In addition, the highest expression level of PeSPL17 occurred at $1 \mathrm{~h}$, while that of PeSPL2 occurred at $24 \mathrm{~h}$. The relative expression of genes PeSPL1, -3, -4, -11, -21, -22, -23, -25, -27, -28, -29 , and 30 peaked at $24 \mathrm{~h}$ after treatment, and PeSPL3 was up-regulated by $>330$-fold compared to the control. Expression of three genes (PeSPL16, -17 and 18) peaked $1 \mathrm{~h}$ after treatment, while expression of PeSPL6, -7, -12, -13 , and 24 also increased and the highest levels were observed at $12 \mathrm{~h}$. The peak expression levels for PeSPL10 and PeSPL26 were observed after GA treatment for 3 and $6 \mathrm{~h}$, respectively.

As shown in Fig. 8 for the simulated drought treatments, transcription of all SPL genes changed in moso bamboo leaves that had been watered with the PEG solution compared to the control. Under PEG treatment, expression of PeSPL3, -4, -6, -8, -9, -10,-11, -12, $-16,-22,-23,-24$ was induced rapidly and peaked at $1 \mathrm{~h}$, whereas expression of PeSPL29 and PeSPL30 peaked at $3 \mathrm{~h}$, and PeSPL13 peaked at $6 \mathrm{~h}$. Interestingly, expression of PeSPL12 was strongly upregulated at $1 \mathrm{~h}$ by $>200$-fold, and then declined under drought treatment. PeSPL5, -15, and -19 were found to be down-regulated at all five time points. Comparing the relative expression patterns of all PeSPL genes revealed that five paralogous 


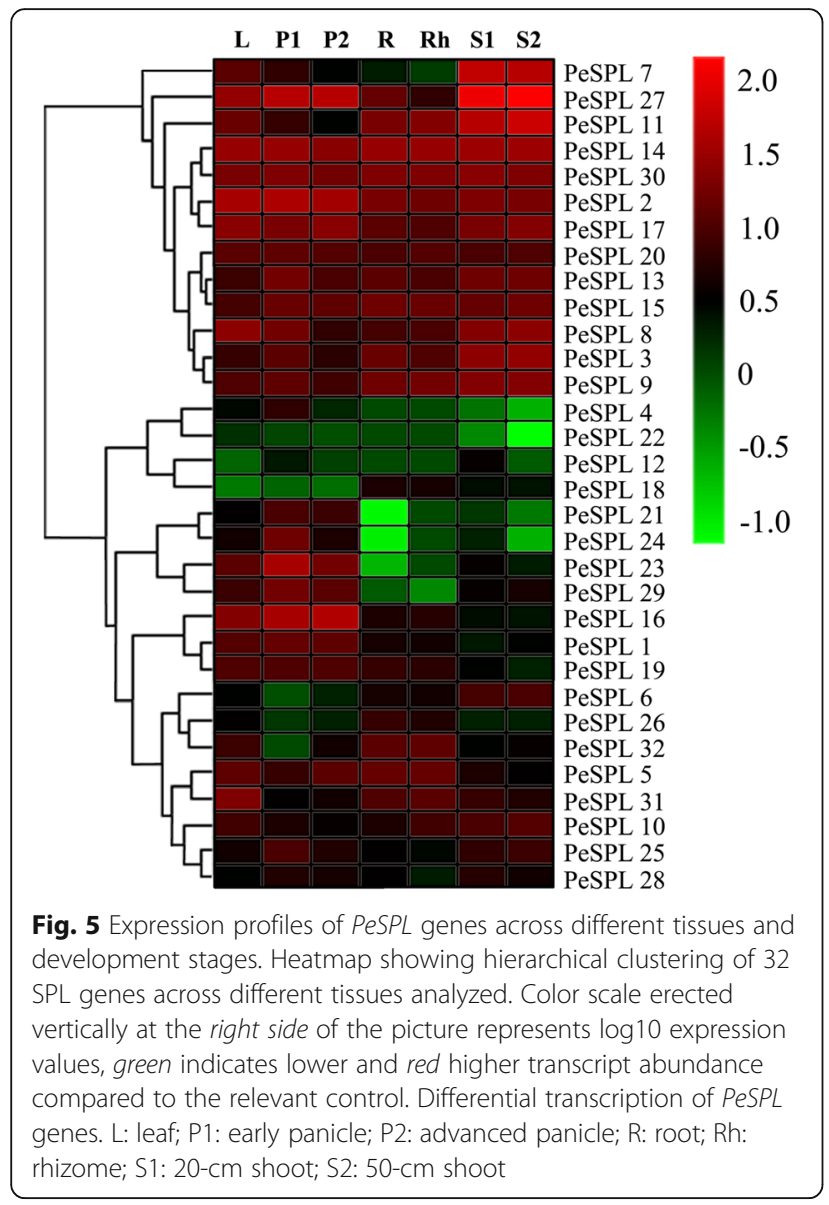

gene pairs (PeSPL4 and -24, PeSPL6 and -25, PeSPL8 and -9, PeSPL10 and -32, and PeSPL12 and -22) showed similar expression patterns in response to drought stress.

To predict possible functions of moso bamboo SPL genes in organ development, we determined the expression profiles of the 32 PeSPLs in five organs; young leaves (L1), mature leaves (L2), roots (R), shoots (S), and panicles $(\mathrm{P})$ using qRT-PCR. There were a variety of transcription patterns observed for the different PeSPLs among the different tissues or developmental stages (Fig. 9). Twenty genes (PeSPL4, -5, -6, -9, -10, $-11,-12,-13,-15,-16,-17,-18,-19,-20,-23,-24$, $-25,-29,-31$, and -32$)$ were expressed in all organs analyzed, while the remaining 12 genes showed dramatically different organ-specific expression in the five organs and developmental stages. Among the 32 moso bamboo SPL genes, four showed the highest mRNA accumulation in the panicle (PeSPL7, -8, -21, and -30), two in roots, shoots, and panicles (PeSPL22 and -27), one in mature leaves and roots (PeSPL1), one in young leaves, roots, and panicles (PeSPL28), one in young leaves and mature leaves (PeSPL3), and one only in young leaves (PeSPL26). In addition, some paralogous gene pairs, such as PeSPL4/-24, PeSPL5/-31, PeSPL6/ -25, PeSPL10/-32, and PeSPL19/-20, showed similar expression patterns. However, most of the remaining gene pairs had very different expression patterns; for instance, PeSPL1 is highly expressed in mature leaves, while its paralog, PeSPL28, is expressed at a much lower level in the same tissue. Through comparisons with the heatmap, we found that most of the PeSPL genes had very similar expression levels in the same organs. The diverse transcription patterns observed in the different organs indicate that SPL genes in moso bamboo may play roles in the development of specific organs.

\section{Discussion}

The SPL genes are a plant-specific transcription factor family with no homologs in bacteria, animals, or humans $[20,71]$. In the present study, we performed a comprehensive analysis of the SPL gene family in Phyllostachys edulis, and the complex function and characteristics of SPL genes have previously been analyzed in model plant species Arabidopsis and rice. In our study, we identified 32 putative SPL genes in the genome of moso bamboo, which is similar to the number found in maize. Based on analysis of the phylogenetic relationships, the predicted PeSPL gene family was divided into eight groups (G1-G8) (Fig. 1). We found that all eight groups included different genes from rice, maize, and moso bamboo, indicating that the SPL genes had diversified prior to the evolutionary diversification of the three species. The phylogenetic tree also showed that the PeSPL genes grouped tightly with OSSPL and $Z m S B P$ genes, which is consistent with the fact that maize and rice diverged from a common ancestor and are also monocots. In addition, the rice SPL14 gene was shown to promote panicle branching and increased rice grain yield in a previous study. Therefore, it is tempting to assume that PeSPL7, the ortholog of OsSPL14 in group 3 and a high expression level in panicle, is involved in the vegetative and reproductive stages in moso bamboo. For the PeSPL genes, analysis of their diverse intron/exon structures and protein motifs will contribute to an understanding of the different roles they play in development and growth. The PeSPL genes from groups 1,4 , and 7 shared similar exon-intron structures within the same phylogenetic clusters, suggesting that the evolutionary relationships of the SBP domains had a great relationship with the changing structures, which is consistent with the situation in rice [20]. The motif sequences and orders were similar for each pair of genes (Fig. 3), which showed that these gene pairs may have similar functions in moso bamboo [35]. However, we noticed that some PeSPL proteins had highly divergent motif patterns. For instance, motif 


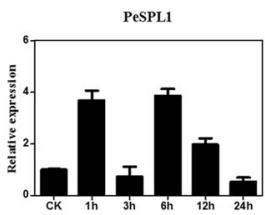

PeSPIS

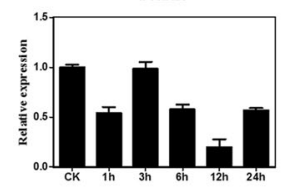

PeSPL9

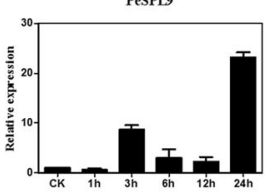

PeSPL13

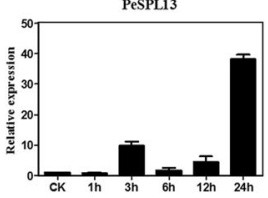

PeSPL17

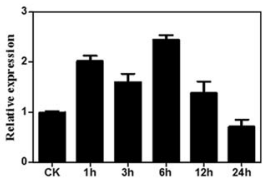

PeSPL

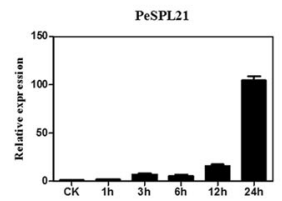

PeSPL25

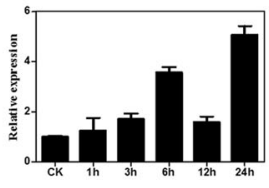

PeSPL29

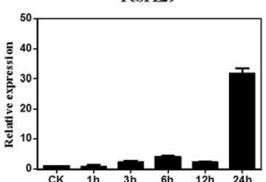

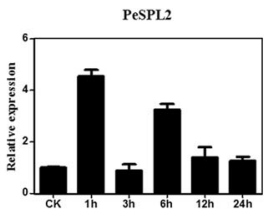

PeSPL6

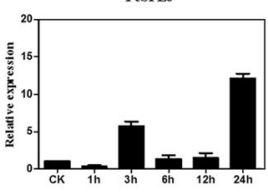

PeSPL10

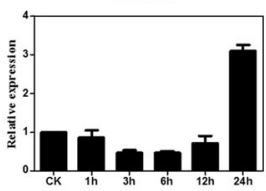

PeSPL14

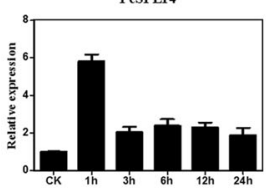

PeSPL18

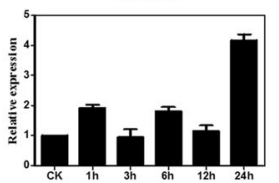

PeSPL22

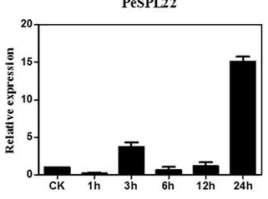

PesPL26

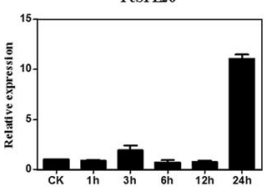

PeSPL30

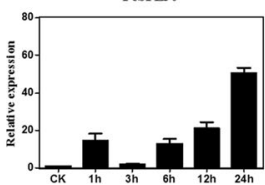

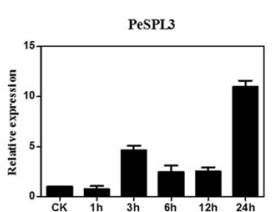

PeSPL?

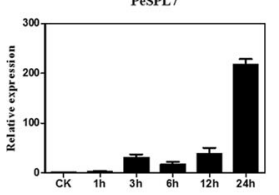

PeSPL11

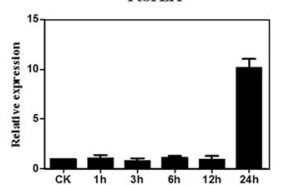

PeSPL15

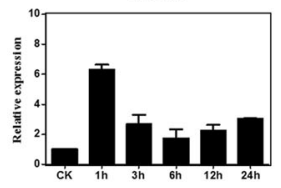

PeSPL19

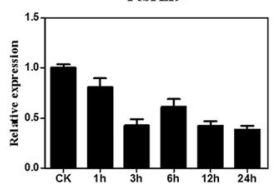

PeSPL23

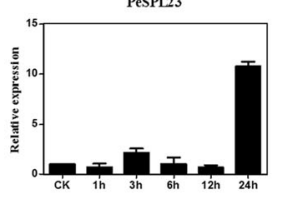

PeSPI27

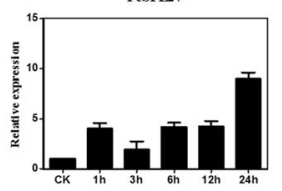

PesPL31

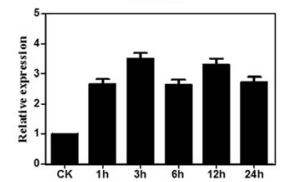

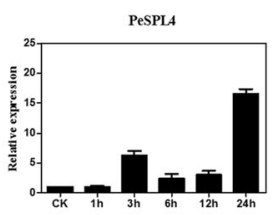

PeSPLS

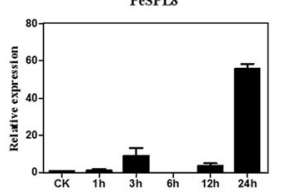

PeSPL12

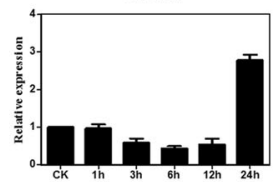

PeSPL16

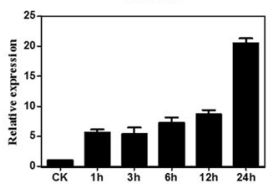

PeSPL20

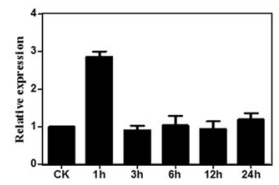

PeSPL24

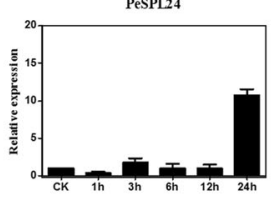

PeSPL28

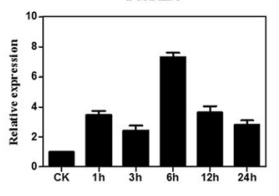

PeSPL32

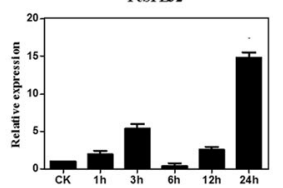

Fig. 6 qRT-PCR expression levels of all PeSPL genes following SA (100 $\mu \mathrm{M})$. The Y-axis indicates the relative expression levels; 0, 1, 3, 6, 9, 12, and 24 (X-axis) indicate hours of treatment. Mean values and standard deviations (SDs) were obtained from three biological and three technical replicates

19 was unique to group 3 and motif 6 was only found in PeSPL2, PeSPL15, and PeSPL17. Motifs 1, 2, and 3 were highly conserved and were present in most PeSPL proteins, which could be important to their functions as transcription factors. Therefore, the different motifs present in the PeSPL proteins are most probably the structural basis for their diverse functions. Many paralogous gene pairs arise from recent gene duplication events, which are important in driving evolution and rapid expansion [72]. In addition, gene duplication events also help organisms adapt to different environments during development and growth [73, 74]. In order to explore the patterns of macroevolution and estimate the evolutionary rates in moso bamboo, we estimated $\mathrm{Ka}$ and $\mathrm{Ks}$ for the paralogous ( $\mathrm{Pe}-\mathrm{Pe}$ ) and orthologous ( $\mathrm{Pe}-\mathrm{Os}$ ) gene pairs and calculated $\mathrm{Ks}$ and the $\mathrm{Ka} / \mathrm{Ks}$ ratios for each gene pair. The Ks values predicted that a large-scale duplication event occurred $\sim 15$ 


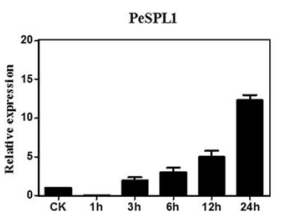

PeSPL5

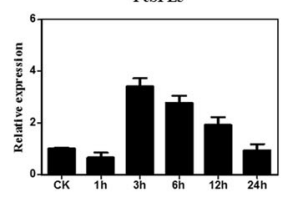

PeSPL9

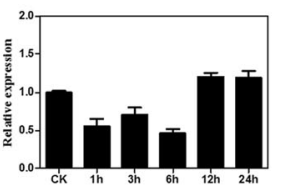

PeSPL13

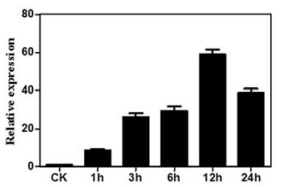

PeSPL17

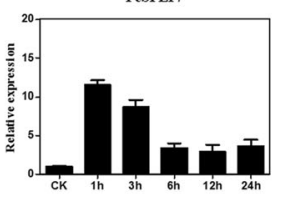

PeSPL21

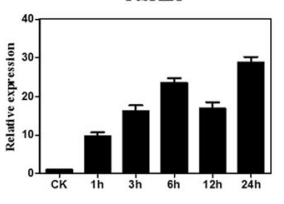

PeSPL25

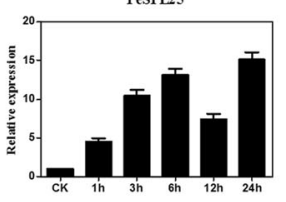

PeSPL29

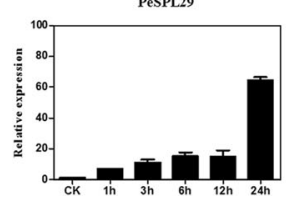

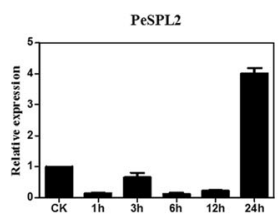

PesPL6

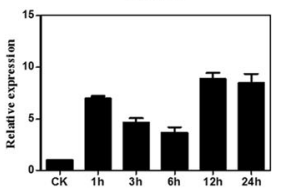

PeSPL10

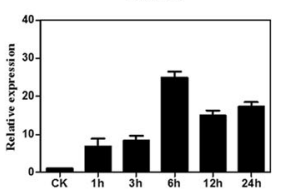

PeSPL14

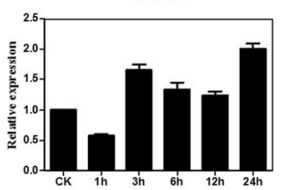

PeSPL18

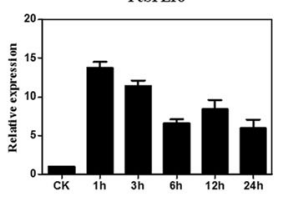

PeSPL22

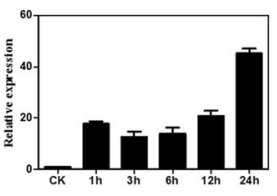

PeSPL26

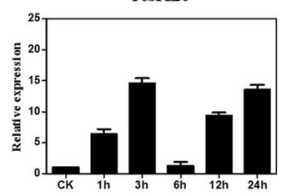

PeSPLI0

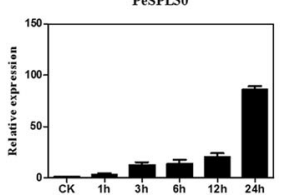

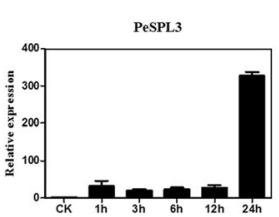

Pespl7

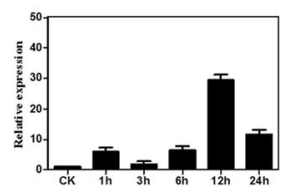

PeSPL11

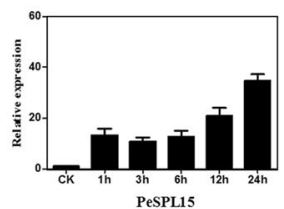

PeSPL15

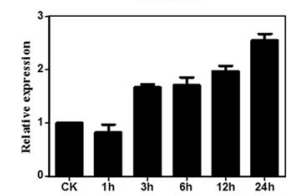

PeSPL19

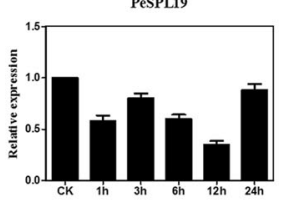

PeSPL23

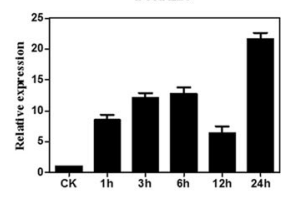

PeSPL27

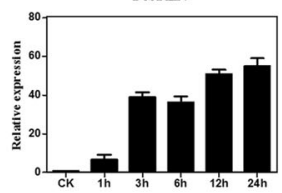

PeSPL11

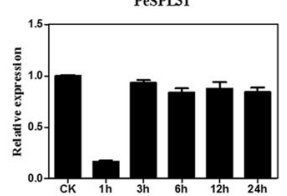

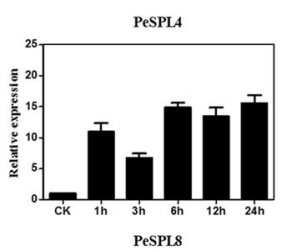

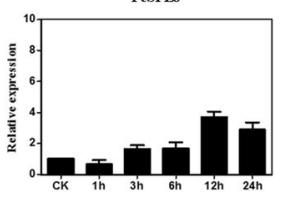

PeSPL12
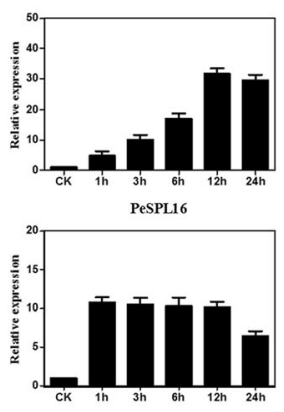

PeSPL20

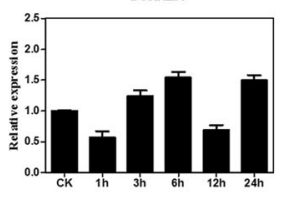

PeSPL24
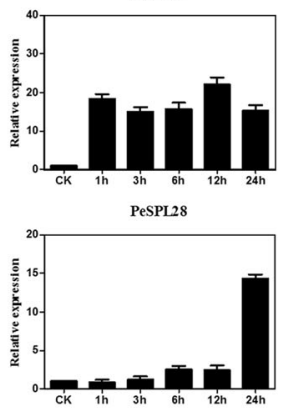

PeSPL 32

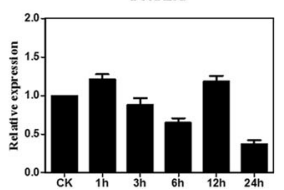

Fig. 7 Expression analysis of PeSPL genes after GA induction. Sampling occurred 0, 1, 3, 6, 9, 12, and 24 h after treatment, and the relative expression levels were analyzed. Untreated sample expression levels $=1$. X-axes represent time points after GA treatment. Y-axes represent relative gene expression values normalized to reference gene TIP41. Bars indicate standard deviations (SD) from three biological replicates

MYA in moso bamboo, and that the divergence times for orthologous gene pairs $(\mathrm{Pe}-\mathrm{Os})$ was approximately 34 MYA. Peng et al. showed that a whole-genome duplication event in moso bamboo occurred 7-12 MYA, and that the divergence time between $P$. edulis and rice was 7-15 MYA [37]. When compared with these results, our study indicates that the SPL gene family experienced an earlier large-scale duplication event and diversified prior to the separation of the two most recent progenitor species. In addition, $\mathrm{Ka} / \mathrm{Ks}$ ratio can be used to measure the historical choice of coding sequences [75]. In general, $\mathrm{Ka} / \mathrm{Ks}$ ratios $>1,=1$, and $<1$ 

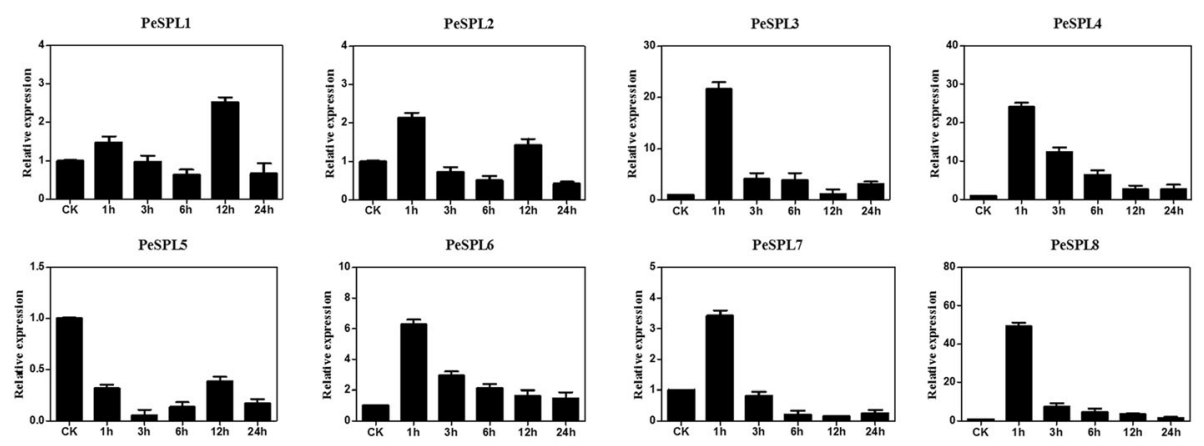

PeSPL9

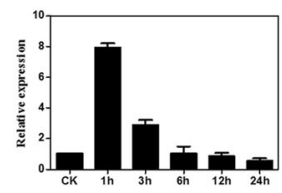

PespL10
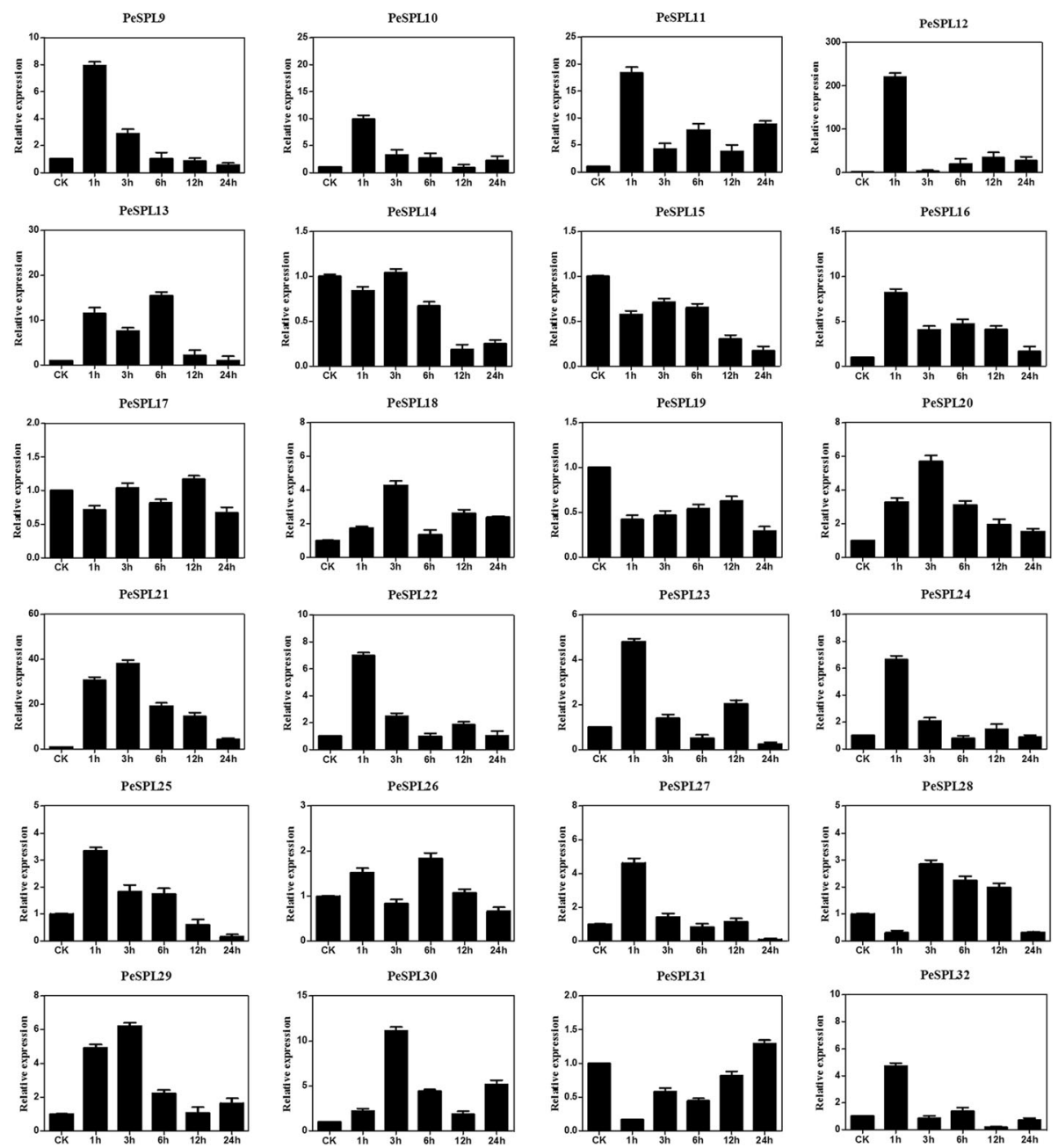

Fig. 8 Expression patterns of all PeSPL genes under drought stress using qRT-PCR. Relative expression levels of 32 SPL genes were examined by qRT-PCR and normalized with respect to the reference gene TIP41 under drought stress treatment. Bars represent standard deviations (SD) of three biological replicates. Y-axes indicate the scale of the relative expression levels. X-axes show time courses of drought stress treatments for each gene

indicate that a gene has experienced positive selection, neutral selection, and negative or purifying selection, respectively $[72,76]$. Interestingly, in our study, the $\mathrm{Ka} /$ $\mathrm{Ks}$ ratios for the $\mathrm{Pe}-\mathrm{Pe}$ and $\mathrm{Pe}-\mathrm{Os}$ gene pairs were large at 0.45 , and imply a strong selection constraint and purifying selection in the PeSPL genes. The analysis of SPL gene expression profiles in different tissues helps us understand the dynamics of gene expression in moso bamboo. Therefore, we used publicly available microarray data (NCBI accession number ERP001341) to analyse the gene expression profiles of the 32 SPL genes in moso bamboo. Our results suggested that most PeSPL genes are expressed at high levels and widely in the different organs or developmental stages that we 


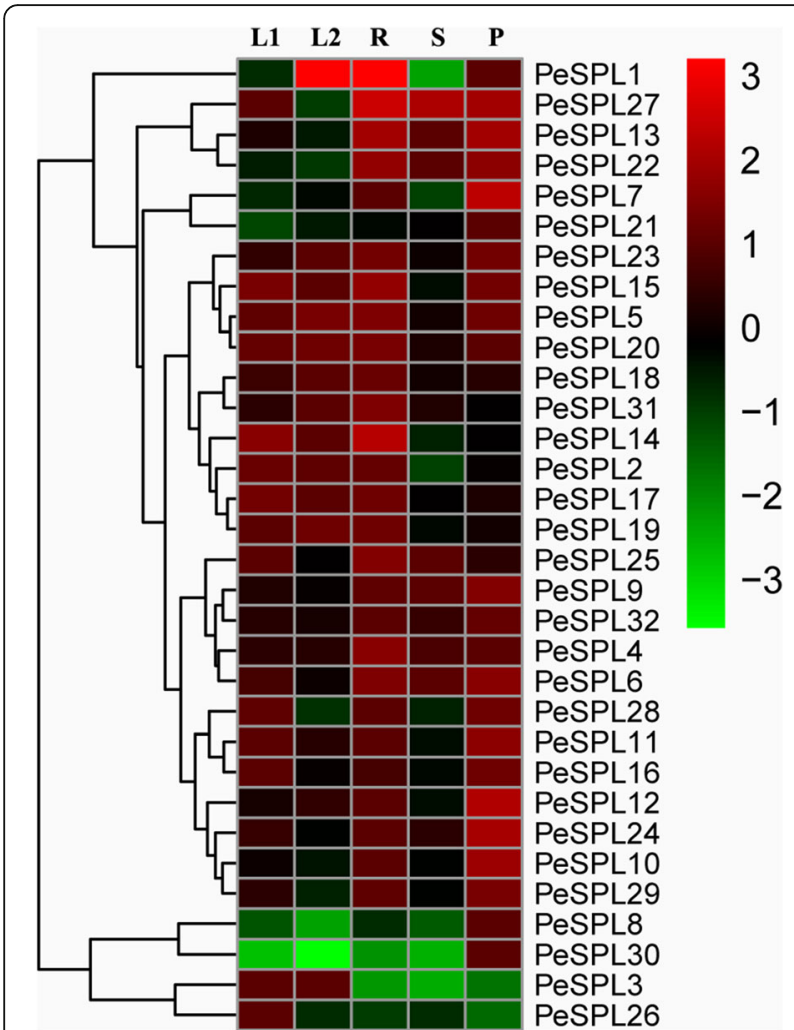

Fig. 9 Expression analysis of PeSPL genes across different tissues and development stages. Sampling from the young leaf, mature leaf, root, shoot and panicle. The green colour indicates lower and red higher transcript abundance compared to the relevant control. L1: young leaf; L2: mature leaf; R: root; S: shoot; P: panicle

examined. For instance, 14 of the 32 genes showed relatively high expression levels in all tissues, demonstrating that they may play important roles in processes involved in moso bamboo development and growth. In addition, we found that several PeSPL genes show tissue-specific expression. The mRNA levels of the 32 $P e S P L$ genes in florets, leaves, and roots were significantly higher than in shoots (Fig. 5), which suggests that PeSPLs might be involved in the development of florets, leaves, and roots in moso bamboo.

Plant genomes contain many stress-related genes allowing plants to adapt to adverse environmental conditions. Previous studies have focused mainly on the function of the SPL gene family in development, but the expression of these genes is rarely studied under stress conditions. In our study, we found that the PeSPL genes are highly regulated by environmental signals and play positive roles in biotic and abiotic stress responses. The PeSPL genes showed significant differential expression patterns under the three biotic and abiotic stress conditions tested. Most PeSPL genes were upregulated by the three stress treatments, suggesting that PeSPL genes may play crucial roles in regulating stress responses in moso bamboo. For example, the high expression of PeSPL7, $-8,-13,-21,-29,-30$ indicated that they may play essential role in response to pathogen infection (SA). In addition, PeSPL3, $-7,-10,-11,-12,-13,-22$, $-27,-29,-30$ and PeSPL3, $-4,-8,-12,-21$ may also have function in response to GA signaling and drought (PEG), respectively. We also found that some genes did not respond to these stresses, especially PeSPL19, which had low expression levels in response to the SA, GA, and PEG treatments. Furthermore, most of the paralogous gene pairs had similar expression levels and patterns under the three stress treatments in the same paralogous pair. These results may indicate that homologous genes have similar functions in the processes of organismal growth and development. Expression profiles of the 32 PeSPLs in five tissues showed that most of these genes were expressed at high levels in all tissues. Previous studies demonstrated that SPLs play vital roles in flower development [71, 77], and miR 156 target the SPL transcription factors that cooperate to regulate bamboo flowering [78]. In our research, we found that most SPL genes in moso bamboo showed higher expression levels in the panicle. Except for PeSPL3 and PeSPL26, all of the PeSPL genes exhibited relatively high transcript levels in the flower (Fig. 9), implying that these genes are important in flower development. In addition, $m i R 156$ target the SPL transcription factors that cooperate to regulate bamboo flowering. Unlike most other plants, flowering is an unpredictable and uncontrollable event in moso bamboo, and the plants die after they flower [2]. The results of our study suggest that expression of PeSPLs in flowers provides a basic understanding for further investigations into the flowering mechanism in moso bamboo.

\section{Conclusions}

The results here represent the first genome-wide analysis of the SPL gene family in moso bamboo. We systematically analyzed the 32 predicted PeSPL genes, including their gene structure, phylogeny, conserved motifs, promoter regions, gene duplication, and expression profiling, which may be related to their biological functions. The phylogenetic analysis clustered these SPL genes into eight groups. In each group, the motif compositions and exon/intron structure were fairly well conserved. Furthermore, the expression patterns of PeSPL genes show that they play potentially important roles in mediating the effects of stress induced by drought (PEG), SA (pathogen infection), and GA. In addition, the expression patterns in various tissues show that PeSPL genes may function in moso bamboo growth and development. The results of our study establish a foundation for future studies on the functions of SPL genes in organ development and the plant stress response, and provide a basic understanding that may allow us to further elucidate the potential functions of the PeSPL genes in moso bamboo. 


\section{Additional file}

Additional file 1: Table S1. Oligonucleotide primers used in qRT-PCR assays for all 32 PeSPL genes. Table S2. Gene names and locus information for the SPL proteins in rice and maize. Table S3. Protein sequences and lengths of the major motifs identified by MEME in the putative PeSPL proteins. Table S4. Nucleotide substitution rates for the paralogous SPL gene pairs identified in the moso bamboo genome. Table S5. Nucleotide substitution rates for the orthologous SPL gene pairs between moso bamboo and rice. Table S6. Summary of abiotic stress-inducible ciselements in the promoter regions of SPL genes in moso bamboo. Table S7. Microarray expression data for the 32 SPL genes in moso bamboo. These primary data was downloaded from NCBI, and then the relative expression level (log10 expression values) of 7 different issues or development stages was obtained after a series of manual processing. $\mathrm{L}$, leaf; P1, early panicle; P2, advanced panicle; R, root; Rh, rhizome; S1, 20-cm shoot; S2, 50-cm shoot. (ZIP $104 \mathrm{~kb}$ )

\section{Abbreviations}

AP1: APETALA1; CDS: Coding sequence; GA: Gibberellin; GDB: Genome database; GSDS: Gene structures display server; Ka: The rate of nonsynonymous substitutions; Ks: The rate of synonymous substitutions; MYA: Million years ago; NCBI: National Center of Biotechnology Information; NCGR: National Center for Gene Research; N-J: Neighbour-Joining; PEG: Polyethylene glycerol; qRT-PCR: Quantitative real-time reverse transcription PCR; RNA-seq: RNA sequencing; SA: Salicylic acid; SBP: SQUAMOSA promoter binding protein; SMART: Simple Modular Architecture Research Tool; SPL: SQUAMOSA promoter binding protein-like; SRA:: Short read archive; TFs: Transcription factors; TIP: Tonoplast intrinsic protein

\section{Acknowledgments}

Special thanks go for the anonymous reviewers for their enlightening comments in reformulating this paper.

\section{Funding}

This study was supported by National Natural Science Foundation of China (No. 31670672) and Project Supported by National Science and Technology Ministry (No. 2015BAD04B03). The funders had no role in the design of the study and collection, analysis, and interpretation of data and in writing the manuscript.

\section{Availability of data and materials}

RNA-seq data for expression profiles from this article were downloaded from NCBI database (accession numbers: ERP001341) (http:/trace.ncbi.nlm.nih.gov/Traces/ sra/?study=ERP001341). The genome sequences of moso bamboo, rice and maize were obtained from moso bamboo GDB server (http://www.bamboogdb.org), Rice Genome Annotation Project database (http://rice.plantbiology.msu.edu) and Phytozome database (https://phytozome.jgi.doe.gov), respectively. Moso bamboo SPL gene IDs were listed in Table 1. The IDs of rice and maize SPL gene were exhibited in Additional file 1: Table S2.

\section{Authors' contributions}

FP designed the experiment, carried out the bioinformatics analyses, and drafted the manuscript. YW participated in the design of the study, implemented the software, and drafted the manuscript. HLL participated in the design of the experiments and helped to draft the manuscript. MW participated in the design of the study, implemented the software, and helped to draft the manuscript. WYC implemented the software, and helped to handle figures and tables. DMC conceived and directed the study, participated in its design and coordination and helped to draft the manuscript. YX, as the correspondence author, provided financial support for the article and designed the way and frame of this study. All authors read and approved the final manuscript.

\section{Ethics approval and consent to participate}

The seeds of moso bamboo were collected from Guilin in Guang Xi Province, China. And the seeds were provided and identified by the Guilin Forestry Bureau. In addition, the tissues of moso bamboo were collected from Ningguo, Anhui Province, China. And the permission of tissues collection and identification for the experiments were obtained from
Jianguo Pei of Ningguo Forestry Bureau. All the materials of moso bamboo used and analyzed were public and available for non-commercial purpose. This article did not contain any studies with human participants or animals performed by any of the authors.

\section{Consent for publication}

Not applicable.

\section{Competing interests}

The authors declare that they have no competing interests.

\section{Publisher's note}

Springer Nature remains neutral with regard to jurisdictional claims in published maps and institutional affiliations.

Received: 10 November 2016 Accepted: 20 June 2017

Published online: 27 June 2017

\section{References}

1. Gui YJ, Sheng W, Quan LY, Zhou CP, Long SB, Zheng HJ, et al. Genome size and sequence composition of moso bamboo: a comparative study. Sci China. 2007:50:700-5.

2. Lin XC, Chow TY, Chen HH, Liu CC, Chou SJ, Huang BL, et al. Understanding bamboo flowering based on large-scale analysis of expressed sequence tags. Genet Mol Res. 2010;9(2):1085-93.

3. Cardon G, Höhmann S, Klein J, Nettesheim K, Saedler H, Huijser P. Molecular characterisation of the Arabidopsis SBP-box genes. Gene. 1999;237:91-104.

4. Klein J, Saedler H, Huijser P. A new family of DNA binding proteins includes putative transcriptional regulators of the Antirrhinum majus floral meristem identity gene SQUAMOSA. Mol Gen Genomics. 1996;250(1):7.

5. Yamasaki K, Kigawa T, Inoue M, Tateno M, Yamasaki T, Yabuki T, et al. A novel zinc-binding motif revealed by solution structures of DNA-binding domains of Arabidopsis SBP-family transcription factors. J Mol Biol. 2004;337: 49-63.

6. Tsubosa $Y$, Sato $H$, Tachimori $Y$, Hokamura N, Hosokawa M, Kinoshita $Y$, et al. Functional analysis of the Arabidopsis thaliana SBP-box gene SPL3: a novel gene involved in the floral transition. Plant J. 1997:12:367-77.

7. Guo AY, Zhu QH, Gu X, Ge S, Yang J, Luo J. Genome-wide identification and evolutionary analysis of the plant specific SBP-box transcription factor family. Gene. 2008:418:1-8

8. Usami T, Horiguchi G, Yano S, Tsukaya H. The more and smaller cells mutants of Arabidopsis thaliana identify novel roles for SQUAMOSA PROMOTER BINDING PROTEIN-LIKE genes in the control of heteroblasty. Development. 2009:136:955-64

9. Gandikota M, Birkenbihl RP, Hohmann S, Cardon GH, Saedler H, Huijser P. The miRNA156/157 recognition element in the 3' UTR of the Arabidopsis SBP-box gene SPL3 prevents early flowering by translational inhibition in seedlings. Plant J. 2007:49(4):683-93.

10. Wu G, Poethig RS. Temporal regulation of shoot development in Arabidopsis thaliana by miR156 and its target SPL3. Development. 2006;133:3539-47.

11. Unte US, Sorensen AM, Pesaresi P, Gandikota M, Leister D, Saedler H, et al. SPL8, an SBP-box gene that affects pollen sac development in Arabidopsis. Plant Cell. 2003;15:1009-19.

12. Xing SP, Salinas M, Hohmann S, Berndtgen R, Huijser P. miR156-targeted and nontargeted SBP-box transcription factors act in concert to secure male fertility in Arabidopsis. Plant Cell. 2010;22(12):3935-50.

13. Jung JH, Seo PJ, Kang SK, Park CM. miR172 signals are incorporated into the miR156 signaling pathway at the SPL3/4/5 genes in Arabidopsis developmental transitions. Plant Mol Biol. 2011:76:35-45.

14. Jung JH, Ju Y, Seo PJ, Lee JH, Park CM. The SOC1-SPL module integrates photoperiod and gibberellic acid signals to control flowering time in Arabidopsis. Plant J. 2012;69:577-88

15. Wang JW, Schwab R, Czech B, Mica E, Weigel D. Dual effects of miR156targeted SPL genes and CYP78A5/KLUH on plastochron length and organ size in Arabidopsis thaliana. Plant Cell. 2008;20(5):1231-43.

16. Yamasaki H, Hayashi M, Fukazawa M, Kobayashi Y, Shikanai T. SQUAMOSA promoter binding protein-like7 is a central regulator for copper homeostasis in Arabidopsis. Plant Cell. 2009:21:347-61.

17. Zhang Y, Schwarz S, Saedler H, Huijser P. SPL8, a local regulator in a subset of gibberellin-mediated developmental processes in Arabidopsis. Plant Mol Biol. 2007:63:429-39. 
18. Lännenpää M, Jänönen I, Hölttä-Vuori M, Gardemeister M, Porali I, Sopanen T. A new SBP-box gene BpSPL1 in silver birch (Betula pendula). Physiol Plantarum. 2004;120:491-500.

19. Kropat J, Tottey S, Birkenbihl RP, Depège N, Huijser P, Merchant S. A regulator of nutritional copper signaling in Chlamydomonas is an SBP domain protein that recognizes the GTAC core of copper response element. P Natl Acad Sci USA. 2005;102(51):18730-5.

20. Xie K, Wu C, Xiong L. Genomic organization, differential expression, and interaction of SQUAMOSA promoter-binding-like transcription factors and microRNA156 in rice. Plant Physiol. 2006;142:280-93.

21. Riese M, Höhmann $S$, Saedler H, Münster T, Huijser P. Comparative analysis of the SBP-box gene families in P. patens and seed plants. Gene. 2007:401: 28-37.

22. Saldarriaga-Noreña H, Waliszewski S, Murillo-Tovar M, Hernández-Mena L, Garza-Rodríguez IDL, Colunga-Urbina E, et al. The maize SBP-box transcription factor encoded by tasselsheath4 regulates bract development and the establishment of meristem boundaries. Development. 2010;137(8): 1243-50.

23. Lu SF, Yang CM, Chiang VL. Conservation and diversity of microRNAassociated copper-regulatory networks in Populus trichocarpa. J Integr Plant Biol. 2011;53(11):879-91.

24. Salinas M, Xing S, Höhmann S, Berndtgen R, Huijser P. Genomic organization, phylogenetic comparison and differential expression of the SBP-box family of transcription factors in tomato. Planta. 2012;235:1171-84.

25. Hou H, Li J, Gao M, Singer SD, Wang H, Mao L, et al. Genomic organization, phylogenetic comparison and differential expression of the SBP-box family genes in grape. PLoS One. 2013;8:e59358.

26. Li J, Hou H, Li X, Jiang X, Yin X, Gao H, et al. Genome-wide identification and analysis of the SBP-box family genes in apple ( Malus $\times$ domestica Borkh.). Plant Physiol Bioch. 2013;70:100-14.

27. Ma Y, Guo JW, Bade R, Men ZH, Hasi A. Genome-wide identification and phylogenetic analysis of the SBP-box gene family in melons. Genet Mol Res. 2014;13:8794-806.

28. Zhang LS, Wu B, Zhao DG, Li CL, Shao FJ, Lu SF. Genome-wide analysis and molecular dissection of the SPL gene family in Salvia miltiorrhiza. J Integr Plant Biol. 2014:56:38-50

29. Zhang SD, Ling LZ. Genome-wide identification and evolutionary analysis of the SBP-box gene family in Castor Bean. PLoS One. 2014;9:e86688.

30. Zhang X, Dou L, Pang C, Song M, Wei H, Fan S, et al. Genomic organization, differential expression, and functional analysis of the SPL gene family in Gossypium hirsutum. Mol Gen Genomics. 2015;290:115-26.

31. Xu Z, Sun L, Zhou Y, Yang W, Cheng T, Jia W, et al. Identification and expression analysis of the SQUAMOSA promoter-binding protein (SBP)-box gene family in Prunus mume. Mol Gen Genet. 2015;290:1701-15.

32. Preston JC, Jorgensen SA, Orozco R, Hileman LC. Paralogous SQUAMOSA PROMOTER BINDING PROTEIN-LIKE (SPL) genes differentially regulate leaf initiation and reproductive phase change in petunia. Planta. 2015;243:42940.

33. Shalom L, Shlizerman L, Zur N, Doronfaigenboim A, Blumwald E, Sadka A. Molecular characterization of SQUAMOSA PROMOTER BINDING PROTEINLIKE (SPL) gene family from Citrus and the effect of fruit load on their expression. Front Plant Sci. 2015;6:389.

34. Li M, Zhao SZ, Zhao CZ, Zhang Y, Xia H, Lopez-Baltazar J, Wan SB, Wang XJ. Cloning and characterization of SPL-family genes in the peanut (Arachis hypogaea L.). Genet Mol Res Gmr. 2015;15 (1).

35. Song A, Gao T, Wu D, Xin J, Chen S, Guan Z, et al. Transcriptome-wide identification and expression analysis of chrysanthemum SBP-like transcription factors. Plant Physiol Bioch. 2016;102:10-6.

36. Zhang HX, Jin JH, He YM, Lu BY, Li DW, Chai WG, et al. Genome-wide identification and analysis of the SBP-box family genes under Phytophthora capsici stress in pepper (Capsicum annuum L.). Front Plant Sci. 2016;7(151): 504.

37. Peng Z, Lu Y, Li L, Zhao Q, Feng Q, Gao Z, et al. The draft genome of the fast-growing non-timber forest species moso bamboo (Phyllostachys heterocycla). Nat Genet. 2013;45(4):456-61

38. Quevillon E, Silventoinen V, Pillai S, Harte N, Mulder N, Apweiler R, et al. InterProScan: protein domains identifier. Nucleic Acids Res. 2005;33:116-20.

39. Thompson JD, Higgins DG, Gibson TJ. CLUSTAL w: improving the sensitivity of progressive multiple sequence alignment through sequence weighting, position-specific gap penalties and weight matrix choice. Nucleic Acids Res. 1994;22:4673-80.
40. Tamura K, Peterson D, Peterson N, Kumar S. MEGA5: molecular evolutionary genetics analysis using maximum likelihood, evolutionary distance, and maximum parsimony methods. Mol Biol Evol. 2011;28:2731-9.

41. Bailey TL, Williams N, Misleh C, Li WW. MEME: discovering and analyzing DNA and protein sequence motifs. Nucleic Acids Res. 2006;34:369-73.

42. Finn RD, Mistry J, Schuster-Bockler B, Griffiths-Jones S, Hollich V, Lassmann T, et al. Pfam: clans, web tools and services. Nucleic Acids Res. 2006;34:D24751.

43. Letunic I, Copley RR, Schmidt S, Ciccarelli FD, Doerks T, Schultz J, et al. SMART 4.0: towards genomic data integration. Nucleic Acids Res. 2004;32: D142-4.

44. Blanc $\mathrm{G}$, Wolfe $\mathrm{KH}$. Widespread paleopolyploidy in model plant species inferred from age distributions of duplicate genes. Plant Cell. 2004;16(7): 1667-78.

45. Altschul SF, Madden TL, Schäffer AA, Zhang J, Zhang Z, Miller W, et al. Gapped BLAST and PSI-BLAST: a new generation of protein database search programs. Nucleic Acids Res. 1997:25(17):3389-402.

46. Guo L, Chen Y, Ning Y, Dai X, Yang W, Yin T. Differential retention and expansion of the ancestral genes associated with the paleopolyploidies in modern rosid plants, as revealed by analysis of the extensins super-gene family. BMC Genomics. 2014:15(1):1-13.

47. Carlini DB, Stephan W. In vivo introduction of unpreferred synonymous codons into the drosophila Adh gene results in reduced levels of $\mathrm{ADH}$ protein. Genetics. 2003;163:239-43.

48. Librado P, Rozas J. DnaSP v5: a software for comprehensive analysis of DNA polymorphism data. Bioinformatics. 2009;25(11):1451-2.

49. Rozas J. DNA sequence polymorphism analysis using DnaSP. Meth Mol Biol. 2009:537:337-50.

50. Lin $Y$, Cheng $Y$, Jin J, Jin $X$, Jiang $H$, Yan $H$, et al. Genome duplication and gene loss affect the evolution of heat shock transcription factor genes in legumes. PLoS One. 2014;9(7):e102825.

51. Cao J, Huang JL, Yang YP, Hu XY. Analyses of the oligopeptide transporter gene family in poplar and grape. BMC Genomics. 2011;12:465.

52. Higo K, Ugawa Y, Iwamoto M, Korenaga T. Plant cis-acting regulatory DNA elements (PLACE) database: 1999. Nucleic Acids Res. 1999;27(1):297-300.

53. Lescot $\mathrm{M}$, et al. PlantCARE, a database of plant cis-acting regulatory elements and a portal to tools for in silico analysis of promoter sequences. Nucleic Acids Res. 2002;30:325-7.

54. Zhang M, Sun H, Fei Z, Zhan F, Gong X, Gao S. Fastq_clean: An optimized pipeline to clean the Illumina sequencing data with quality control. In: Bioinformatics and Biomedicine (BIBM), 2014 IEEE International Conference on: 2014. New York: IEEE; 2014. p. 44-8.

55. Trapnell C, Roberts A, Goff L, Pertea G, Kim D, Kelley DR, et al. Differential gene and transcript expression analysis of RNA-seq experiments with TopHat and cufflinks. Nat Protoc. 2012;7:562-78.

56. Toufighi K, Brady SM, Austin R, Ly E, Provart NJ. The botany array resource: e-Northerns, expression angling, and promoter analyses. Plant J. 2015;43: 153-63.

57. Hui M, Lin F, Zhu C, Xue C, Zhao H, Yan X. Genome-wide identification and expression analysis of the IQD gene family in Populus trichocarpa. Plant Sci. 2014:229:96-110.

58. Schmittgen TD, Livak KJ. Analyzing real-time PCR data by the comparative C(T) method. Nat Protoc. 2008:3:1101-8.

59. Wu H, et al. Genome-wide analysis of the AP2/ERF transcription factors family and the expression patterns of DREB genes in moso bamboo (Phyllostachys edulis). PLoS One. 2015;10(5):e0126657.

60. Saeed Al, Bhagabati NK, Braisted JC, Wei L, Sharov V, Howe EA, et al. TM4 microarray software suite. Method Enzymol. 2006;411:134-93.

61. Le DT, Nishiyama R, Watanabe $Y$, Vankova R, Tanaka M, Seki M, et al. Identification and expression analysis of cytokinin metabolic genes in soybean under normal and drought conditions in relation to cytokinin levels. PLoS One. 2012;7(8):e42411.

62. Walther $D$, Brunnemann $R$, Selbig J. The regulatory code for transcriptional response diversity and its relation to genome structural properties in $A$. thaliana. PLoS Genet. 2007;3:216-29.

63. Fang $Y$, You J, Xie $K$, Xie W, Xiong L. Systematic sequence analysis and identification of $\mathrm{t}$ issue-specific or stress-responsive genes of NAC transcription factor family in rice. Mol Gen Genomics. 2008;280:547-63.

64. Jian W, Zhen P, Liu S, He Y, Lin C, Kong F, et al. Genome-wide analysis of Aux/IAA gene family in Solanaceae species using tomato as a model. Mol Gen Genet. 2012;287:295-311. 
65. Schuster SC. Next-generation sequencing transforms today's biology. Nat Methods. 2008;5(1):16-8.

66. Metzker ML. Sequencing technologies-the next generation. Nat Rev Genet. 2010;11(1):1-13.

67. Gao J, Zhang Y, Zhang C, Qi F, Li X, Mu S, et al. Characterization of the floral transcriptome of Moso bamboo (Phyllostachys edulis) at different flowering developmental stages by transcriptome sequencing and RNA-Seq analysis. PLoS One. 2014;9(6):e98910.

68. Gao ZM, Li CL, Peng ZH. Generation and analysis of expressed sequence tags from a normalized CDNA library of young leaf from ma bamboo ( Dendrocalamus latiflorus Munro). Plant Cell Rep. 2011;30(11):2045-57.

69. Peng Z, Lu T, Li L, Liu X, Gao Z, Tao H, et al. Genome-wide characterization of the biggest grass, bamboo, based on 10,608 putative full-length CDNA sequences. BMC Plant Boil. 2010;10(1):1-13.

70. Skirycz A, Inzé D. More from less: plant growth under limited water. Curr Opin Biotech. 2010;21(2):197-203.

71. Cardon G, Höhmann S, Nettesheim K, Saedler H, Huijser P. Functional analysis of the Arabidopsis thaliana SBP-box gene SPL3: a novel gene involved in the floral transition. Plant J. 1997;12:367-77.

72. Cannon SB, Mitra A, Baumgarten A, Young ND, May G. The roles of segmental and tandem gene duplication in the evolution of large gene families in Arabidopsis thaliana. BMC Plant Boil. 2004;4(1):10.

73. Bowers JE, Chapman BA, Rong J, Paterson AH. Unravelling angiosperm genome evolution by phylogenetic analysis of chromosomal duplication events. Nature. 2003;422(6930):433-8.

74. Gu ZL, Steinmetz LM, Gu X, Li WH. Role of duplicate genes in genetic robustness against null mutations. Nature. 2003:421:63-6.

75. Wu M, Li Y, Chen D, Liu H, Zhu D, Xiang Y. Genome-wide identification and expression analysis of the IQD gene family in moso bamboo (Phyllostachys edulis). Sci Rep-UK. 2016;6:24520.

76. Shiu SH, Karlowski WM, Pan R, Tzeng YH, Mayer KF, Li WH. Comparative analysis of the receptor-like kinase family in Arabidopsis and rice. Plant Cell. 2004;16:1220-34

77. Manning K, Tör M, Poole M, Hong Y, Thompson AJ, King GJ, et al. A naturally occurring epigenetic mutation in a gene encoding an SBP-box transcription factor inhibits tomato fruit ripening. Nat Genet. 2006;38:948-52.

78. Gao J, Wei G, Zhang Y, et al. Identification and characterization of microRNAs at different flowering developmental stages in moso bamboo (Phyllostachys edulis) by high-throughput sequencing. Mol Gen Genomics. 2015;290(6):2335-53.

\section{Submit your next manuscript to BioMed Central and we will help you at every step:}

- We accept pre-submission inquiries

- Our selector tool helps you to find the most relevant journal

- We provide round the clock customer support

- Convenient online submission

- Thorough peer review

- Inclusion in PubMed and all major indexing services

- Maximum visibility for your research

Submit your manuscript at www.biomedcentral.com/submit

) Biomed Central 